\title{
A computational model of peridynamic theory for deflecting behavior of crack propagation with micro- cracks
}

\author{
Muhammed Fatih Basoglu', Zihni Zerin', Adnan Kefal ${ }^{2, *}$, and Erkan Oterkus ${ }^{3}$ \\ 1 Department of Civil Engineering, Ondokuz Mayis University, Samsun 55139, Turkey \\ 2 Faculty of Naval Architecture and Ocean Engineering, Istanbul Technical University, Maslak- \\ Sariyer, 34469 Istanbul, Turkey \\ 3 Department of Naval Architecture, Ocean and Marine Engineering, University of Strathclyde, \\ Glasgow G4 0LZ, United Kingdom \\ * Correspondence: kefaladnan@itu.edu.tr
}

\begin{abstract}
The critical effect of micro level defects should be examined at macro level to better understand the fracture behaviors of engineering materials. This study investigates the branching and deflecting behavior of a macro (main) crack in presence of multiple number of micro-cracks at the vicinity of the crack tip. For this purpose, a non-local continuum theory, known as Peridynamics (PD), is utilized based on the original set of bond-based PD equations. The main advantage of using PD is its characteristic superiorities on the modelling of dynamical fracture. Various example problems with inclined-linear and/or curvilinear micro-crack clusters are solved through the implantation of different numerical models to better understand the micro-crack toughening mechanisms. After validating the PD implementation with a benchmark case, several combinations of multiple micro-cracks with various locations are considered. To capture complex forms of crack branches, the positions of micro-cracks are designated to follow an encircling and spreading patterns at the vicinity of the main-crack tip. Hence, more internal energy is dissipated through the generation of new crack surfaces such that the main-crack deflects along a more twisting path. It has been observed that depending on the amount of dissipated energy, the propagation speed of main-crack alters. Also, it has been demonstrated that encircling potential crack propagation regions with micro-cracks provides an augmented toughness to the brittle materials. Overall, the efficiency and robustness of the PD theory are revealed for simulating crack propagation in brittle materials.
\end{abstract}

Keywords: Peridynamics, micro cracks, interactions of cracks, material toughness, crack propagation, crack curving and branching. 


\section{Introduction}

Most of the today's engineering structures including civil, marine, and aerospace applications suffer from the initiation and propagation of defects and/or cracks either at micro/macro level. This type of phenomena leads to the failure of the structural components and thus may break down the operational excellence of the sub-structural systems in many incidents. For instance, brittle materials such as concrete, ceramics, and rocks endow with some superior mechanical properties like hardness, wear resistance, and oxidation resistance. On the other hand, their unfavourable features, like low-toughness and high-brittleness, considerably limit their engineering applications. Therefore, investigating and enhancing the fracture toughness of the brittle materials have been a noteworthy subject for various researchers [1-3].

The micro-cracks and/or voids may have already been existing in brittle materials. Otherwise, these defects can be easily formed due to high stress concentrations. Therefore, this kind of micro-defects are inevitable in the brittle materials [4]. Nevertheless, several studies [5, 6] indicated that the presence of micro-cracks may increase the material toughness, which is referred as the phenomenon of "micro-crack toughening". Besides, in the presence of a maincrack, the crack propagation behavior is significantly influenced by the parameters such as density, location and orientation of micro-cracks, since the stress intensity of the main-crack alters correspondingly [3, 7-9]. However, non-uniform distribution of micro-defects may lead to complex mechanical behavior, which cannot be readily predicted with the use of classical fracture mechanics. For example, various classical approaches have been used to investigate only the uniform distribution of micro-cracks over the last few decades [10-14].

In analytical approaches, the effects of micro-defects are defined by degrading elastic constants based on homogenization methodologies $[15,16]$. To define the mechanical behavior of the damaged region, these homogenization techniques utilize the characteristic features of micro-defects such as orientation, density, and shape of the defect cluster [6, 17]. Some modified versions of the finite element method (FEM) such as element-erosion, cohesive-zone, and extended-FEM (X-FEM) models have been used as numerical approaches for modelling crack propagation. The crack propagation path is restricted to the element boundaries for the element-erosion and the cohesive-zone techniques, which leads to obtain inaccurate results [18]. On the other hand, the XFEM method can model the arbitrary crack propagation without re-meshing process [19-24]. However, the tuning between input and output fracture energy is required for performing accurate XFEM analysis of dynamic brittle fractures [18, 25]. Also, there are some other drawbacks encountered in the XFEM crack modelling such as dependency 
to the level set methods, inefficiency for predicting crack nucleation and initiation, and requirement of external techniques for simulating crack branching [25]. Thus, a more robust and capable approach is required for determining the mechanical behavior of materials in terms of being capable of predicting crack nucleation, propagation, and interactions. These shortcomings of classical numerical approaches are circumvented with the introduction of Peridynamics (PD) [26] which naturally satisfies all necessities for modelling, nucleating, and propagating cracks in a continuum.

$\mathrm{PD}$ is a non-local form of classical continuum mechanics (CCM), where the divergence of stress term is calculated through integro-differential equations defined as the integration of the force densities acting between material points (particles) over an internal length parameter, known as the horizon of each particle. Since spatial derivatives of stress quantities are eliminated in this way, the PD equations become valid even at the discontinuous regions of the continuum [27]. The initial version of the PD theory is called as bond-based PD theory [26] where the Poisson's ratio is restricted to the values of $1 / 4$ and $1 / 3$ in three- and two-dimensional structures, respectively. This limitation is eliminated by the introduction of the state-based PD theory [28], which is a generalization of the bond-based PD theory. Various research studies have been conducted to demonstrate the efficiency and robustness of the PD theory as well as its mechanics-related applications to different class of materials. For example, a convergence study was made between PD force densities and forces of the classical elasticity [29]. Also, fiber-reinforced composite materials under thermal and mechanical loadings were investigated with employing PD theory [30]. Moreover, a novel PD model was proposed [31] for anisotropic materials. Besides, couplings of PD and FEM methods were applied for failure prediction [32, 33]. In addition, the fracture characteristics of polycrystalline and multiscale materials were analyzed by using PD theory $[34,35]$. Furthermore, heat conduction and thermo-mechanics issues were studied within the framework of PD theory [36, 37]. More recently, a PD plasticity model was presented based on von Mises yield criteria and isotropic hardening [38]. Overall, the comprehensive details of the theory and its detailed literature survey can be found in the recent book of Madenci and Oterkus [39].

In this study, we investigate various PD implementations for simulating branching and deflecting propagation of a main-crack in the presence of multiple inclined-linear and/or curvilinear micro-cracks in brittle materials. Up until now, the interactions of cracks have been widely investigated for the uniform micro-crack-patterns involving limited number of cracks $[6,9,11]$. To the best of authors knowledge, although the fundamental mechanisms of this 
aspect have been discussed, the studies related to more complex crack patterns with numerous micro-defects is not widely available in the current literature [10]. Most recently, Vazic et al. [40] presented a PD model for crack propagation and interactions between a main-crack and a parallel micro-crack cluster. Nevertheless, the positioning patterns of micro-cracks were placed well-ordered, which were selected as parallel to the main-crack [40]. We have extended these cases based on bond-based PD models to simulate the mechanical behavior of arbitrary microcrack patterns having various orientation with respect to the main-crack, which has been done for the first time in the literature. Considering the importance of materials design on amplification and shielding effect, this study presents viable cases for the understanding of material toughness of brittle materials involving micro-cracks with varying positions and orientations. The main novelty of the current study is to propose a new schematic of microcrack distribution around the main-crack tip by using PD theory. According to the results, it has been observed that the greatest shielding effect can be achieved through allocating a crescentlike micro-crack distribution behind the main-crack tip. Hence it leads to a significant increase in the material toughness. This paper is organized as follows. The bond-based PD theory is briefly described in Section 2. Then, the numerical implementation of the theory and results are presented in Section 3. Finally, some concluding remarks are drawn in Section 4.

\section{The formulation of Peridynamic theory}

The CCM theory cannot deal with cracks since its governing equations are partial differential equations (PDEs), which are indefinite at the discontinuities. In order to overcome this problem, mechanics of continuous and discontinuous media are unified within a set of PD equations by replacing the PDEs with integral equations [27]. Hence, the PD equations are valid everywhere in a body regardless of discontinuities [34]. PD theory was first introduced by Silling [27]. The fundamental assumption of the theory is that the force between the two particles is only a function of the positions of these particles and their relative displacement. This initial stage of the PD theory was called "bond-based PD theory", after a generalized version, i.e., "state-based PD theory", was revealed by Silling et al. [28].

Consider that any two particles of the material are interacting with each other, such as particles at $\mathbf{x}$ and $\mathbf{x}^{\prime}$ locations. At initial undeformed case, the relative position vector (with respect to the $\mathbf{x}$ ) between the particles is $\xi=\mathbf{x}^{\prime}-\mathbf{x}$, which is also called as reference-bond. After the deformation, the displacement vectors of the particles $\mathbf{x}$ and $\mathbf{x}^{\prime}$ are defined as $\mathbf{u}(\mathbf{x}, t)$ and $\mathbf{u}\left(\mathbf{x}^{\prime}, t\right)$, respectively. The difference between these two displacement vectors provides the 
change in the length of the reference-bond, which can be stated as $\boldsymbol{\eta}=\mathbf{u}\left(\mathbf{x}^{\prime}, t\right)-\mathbf{u}(\mathbf{x}, t)$ (Figure 1). Therefore, the current relative position vector, $\xi+\boldsymbol{\eta}$, defines the deformed-bond between the particles [26, 39]. The bond-stretch between the particles can be expressed as:

$$
s=\frac{|\xi+\eta|-|\xi|}{|\xi|}
$$

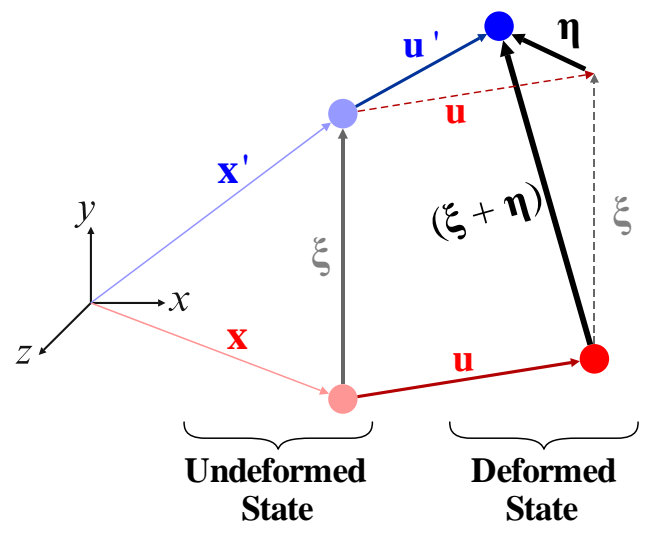

Figure 1 Kinematics of two material particles

According to the bond-based PD theory, the idea of bond interaction between any two particles can be considered as an elastic spring (linear or nonlinear) for elastic materials. Hence, the energy in a bond due to the relative displacement vector $\boldsymbol{\eta}$ only through the scalar distance is defined as a scalar micro-potential function, $w$ (Figure 4):

$$
w(\boldsymbol{\eta}, \xi)=\frac{1}{2} c s^{2}|\xi|
$$

where $c$ represents the bond-constant.

The bond-force, $\mathbf{f}$, can be derived from this scalar valued micro-potential (Figure 2) as $[26,27,39]:$

$$
\mathbf{f}(\boldsymbol{\eta}, \xi)=\frac{\partial w}{\partial \boldsymbol{\eta}}(\boldsymbol{\eta}, \xi)=\frac{\xi+\boldsymbol{\eta}}{|\xi+\boldsymbol{\eta}|} f(|\xi+\boldsymbol{\eta}|, \xi) \quad \forall \boldsymbol{\eta}, \boldsymbol{\xi}
$$

where $f$ is a scalar-valued function of the bond-constant, $c$, and the bond-stretch, $s$, is defined as:

$$
f(|\xi+\boldsymbol{\eta}|, \xi)=c s
$$




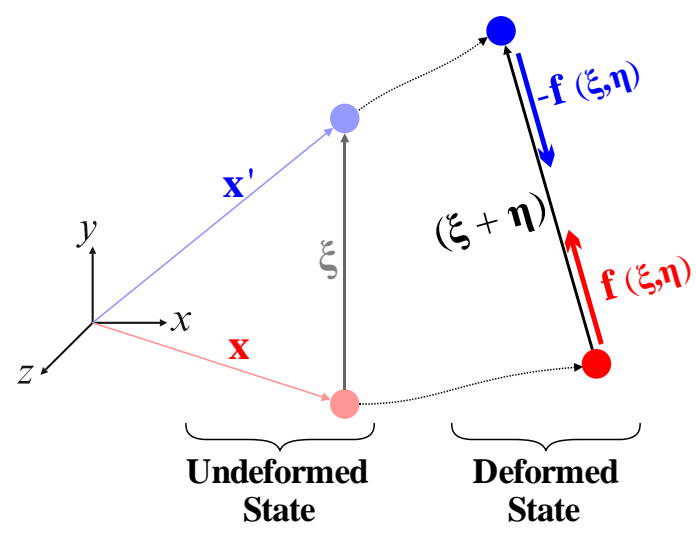

Figure 2 Bond-based PD force function

Herein, it is assumed that these interactions between any two particles in a material are restricted to an internal length parameter, $\delta$, called horizon-radius (Figure 3). In other words, a particle cannot establish a bond with another particle beyond this horizon (expressed as $H$ ) as [26]:

$$
|\xi|>\delta \Rightarrow \mathbf{f}(\xi, \boldsymbol{\eta})=0 \quad \forall \boldsymbol{\eta}
$$

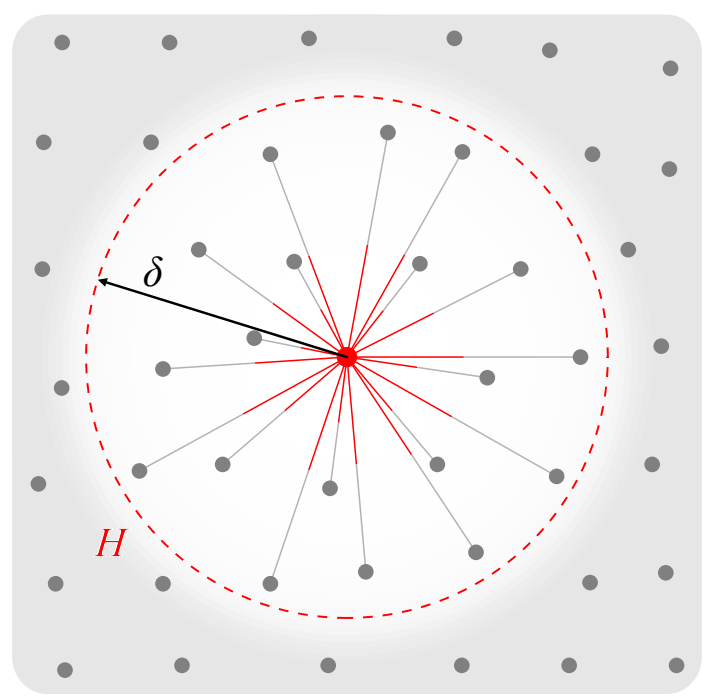

Figure 3 Interactions of a material particle with the particles in its horizon

The micro-potential, $w$, given in Eq. (2) is a micro-elastic based potential energy of a bond in a continuum. As is defined, a particle has bond-interactions as many as the number of particles in its horizon, $H$. Integration of these potential energies on a particle is expressed as strain-energy-density, $W$, and can be obtained by summing the half of the micro-potentials, $w$ , in each bond [26, 39] (Figure 4), as:

$$
W=\frac{1}{2} \int_{H} w d H=\frac{1}{2} \int_{H}\left(\frac{1}{2} c s^{2}|\xi|\right) d H
$$



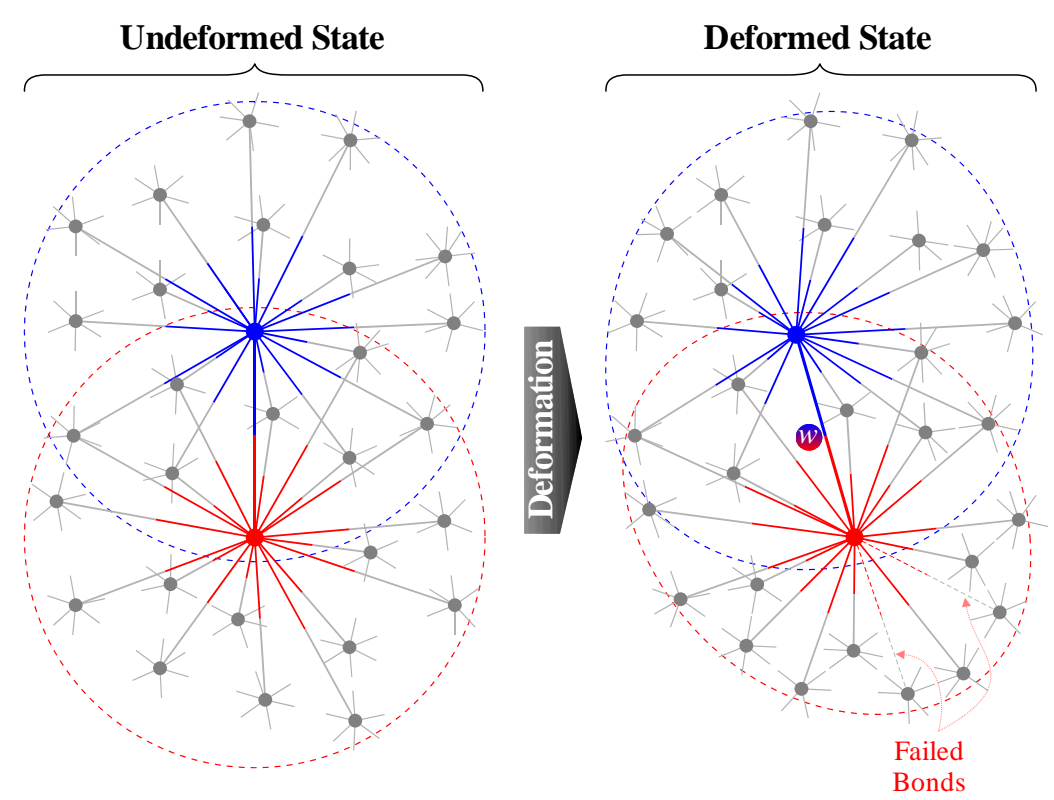

Figure 4 Schematics of PD particles in the undeformed and deformed states

Based on the bond-based PD theory, the bond-constant, $c$, can be obtained in terms of elastic modulus, $E$, thickness, $h$, and horizon-radius, $\delta$, by equating the strain energy densities of the PD and CCM theories for a uniform expansion loading condition in a media as [26, 39]:

$$
c=\frac{9 E}{\pi h \delta^{3}} \text { (for two-dimensional structures) }
$$

The bond-based PD theory establishes the equation of motion of a particle as [26]:

$$
\rho \ddot{\mathbf{u}}(\mathbf{x}, t)=\int_{H} \mathbf{f}\left(\mathbf{u}\left(\mathbf{x}^{\prime}, t\right)-\mathbf{u}(\mathbf{x}, t), \mathbf{x}^{\prime}-\mathbf{x}\right) d V^{\prime}+\mathbf{b}(\mathbf{x}, t)
$$

where $\mathbf{b}(\mathbf{x}, t), \ddot{\mathbf{u}}(\mathbf{x}, t), \rho$, represents the body force, acceleration, and density of particle located at $\mathbf{x}$, respectively. In Eq. (8), the symbol $d V^{\prime}$ denotes the infinitesimally small volume of material points located at position $\mathbf{x}^{\prime}$. In order to solve the Eq. (8), the continuum can be discretized into material-points (with finite volumes) at the initial undeformed case. If the displacement of material point $k$ at time step $n$ is expressed as $\mathbf{u}_{k}^{n}$, and the integral is replaced by a finite sum in the discretized form, the equation of motion takes the final form as:

$$
\rho_{k} \ddot{\mathbf{u}}_{k}^{n}=\sum_{j=1}^{N} \mathbf{f}\left(\mathbf{u}_{j}^{n}-\mathbf{u}_{k}^{n}, \mathbf{x}_{j}-\mathbf{x}_{k}\right) V_{j}+\mathbf{b}_{k}^{n}
$$

where $N$ is the number of material-points inside the horizon coverage of the material point $\mathbf{x}_{k}$ , and $V_{j}$ represents the volume of the material point $\mathbf{x}_{j}[26,40]$. Also, in the same manner, the strain-energy-density, $W_{k}$, of material point $k$ at time step $n$ could be discretized as: 


$$
W_{k}^{n}=\frac{1}{2} \sum_{j=1}^{N} w_{k j}^{n} V_{j}
$$

A linear-elastic brittle-material behavior can be observed from the plotted relationship between bond-force, $\mathbf{f}$, and bond-stretch, $s$, in Figure 5. Otherwise stated, when the bondstretch, $s$, exceeds the critical-stretch value, $s_{0}$, the bond interaction between the materialpoints vanish (Figure 4). As is seen, introducing failure is quite simple in PD. Herewith, both crack initiation and propagation can be modelled readily without needing any initial defects $[26,39]$.

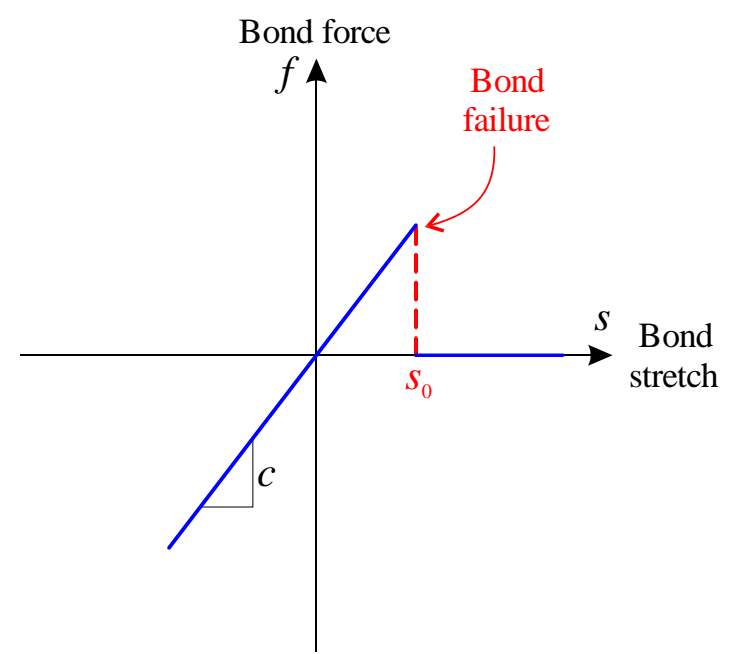

Figure 5 Model for bond failure

For defining this failure formation, a history-dependent scalar-valued failure-function can be written as a factor of the scalar bond-force expression (Eq.4):

$$
f(|\xi+\eta|, \xi)=\operatorname{cs} \mu(t, \xi)
$$

where $\mu(t, \xi)$ is a step function that defines the failure of each interaction as:

$$
\mu(t, \xi)= \begin{cases}1 & \text { if } \quad s\left(t^{\prime}, \xi\right)<s_{0} \text { for all } 0 \leq t^{\prime} \leq t \\ 0 & \text { otherwise }\end{cases}
$$

Due to the bond elimination, a local damage parameter can be defined based on the weighted ratio of the number of the eliminated bonds to the total initial bonds of the related material point as [26, 32]:

$$
\varphi(\mathbf{x}, t)=1-\frac{\int_{H} \mu(\mathbf{x}, t, \xi) d V^{\prime}}{\int_{H} d V^{\prime}}
$$




\section{$3 \quad$ Numerical examples}

In this section, the results of interactions between a main-crack and multiple micro-cracks in a brittle material are presented by utilizing bond-based peridynamic model. First of all, a benchmark problem solved by Vazic et al. [40] is revisited herein to compare and validate the accuracy of the presented PD implementation for interaction micro-cracks with a main-crack. Then six different case studies are designed and analyzed by including multiple micro-cracks with various type of orientations and positions. The main aim of these cases is to demonstrate the deflecting behavior of main-cracks in the presence of micro-cracks having inclined-linear and/or curvilinear patterns.

As shown in Figure 6, a pre-cracked Poly (methyl-methacrylate) square-plate with the size of $L \times L=50 \times 50 \mathrm{~mm}^{2}$ is subjected to a constant velocity of $\mathrm{v}=5 \mathrm{~m} / \mathrm{s}$ at the bottom and top edges of the plate. This load represents a displacement-controlled tensile-load and kept constant throughout the PD simulation. The length of the main-crack is $L_{m}=25 \mathrm{~mm}$ and the properties of the Poly material, i.e., acrylic glass, is given in Table 1. Note that actual Poisson's ratio of the acrylic glass is a slightly higher than the Poisson's ratio corresponding to bond-based PD assumptions. However, since this small variation has a negligible influence on the speed or propagation of cracks for dynamic fracture problems [41], the value of $v=1 / 3$ can be safely used in the following PD analysis.

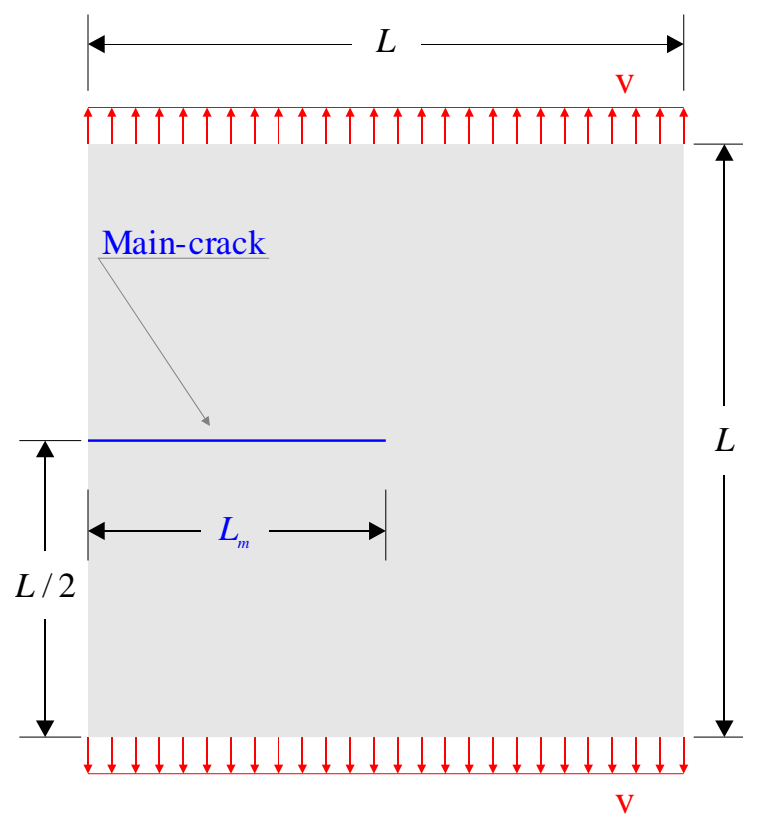

Figure 6 Problem setup 
Table 1 Material properties of Poly (methyl methacrylate)

\begin{tabular}{cccc}
\hline $\begin{array}{c}\text { Density } \\
\rho\end{array}$ & Young's modulus & Poisson ratio & Fracture energy \\
$G_{0}$
\end{tabular}

Spatial discretization of the model is generated with a single layer of $(500 \times 500 \times 1)$ material-points by using meshless scheme. According to the discretization, the grid size is specified as $\Delta x=10^{-4} \mathrm{~m}$ and the horizon-radius is defined as $\delta=3.015 \Delta x$. Moreover, the time step size is determined as $\Delta t=4 \cdot 10^{-8} \mathrm{~s}$, which is less than the stable-time-step size [26], and the critical-stretch value is determined as $s_{0}=0.030857$ [40]. To be able to apply the velocity boundary conditions on the edges of the plate, virtual material points are generated with the total depth of $\delta$ [39] and added on these boundaries as depicted in Figure 7.

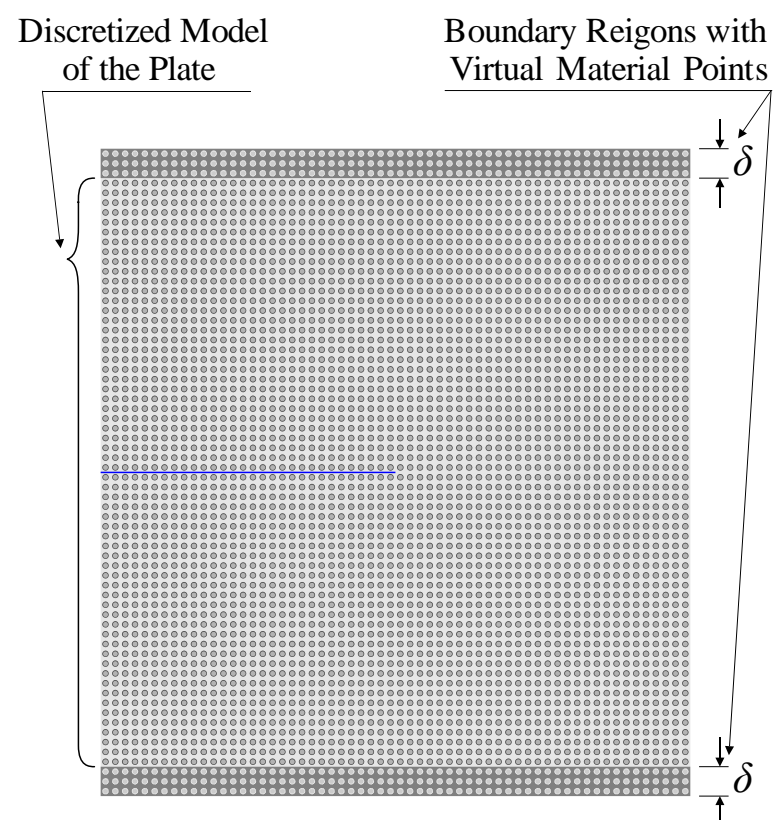

Figure 7 Discretization of PD model

\subsection{Benchmark problem}

In the benchmark case, two micro-cracks with the size of $2 l$ are placed as symmetric and parallel with respect to the main-crack as shown in Figure 8 . The length parameter, $l$, is defined by the size of plate edge as $l=L / 100$. For the purpose of results comparison with the reference [40], a horizontal position parameter, $d$, is defined according to the main-crack tip as depicted in Figure 8. Note that the vertical positions of the micro-cracks are fixed to the value of $h=1.25 l$. The crack patterns for varying values of $d=-2 l, 0,2 l$ are presented in Figure 9, which demonstrates that our results are very analogous to the crack patterns observed in the 
reference [40]. Hence, these benchmark results clearly confirm that our bond-based PD implementation is suitable to predict the response of a main-crack in the presence of microcracks.

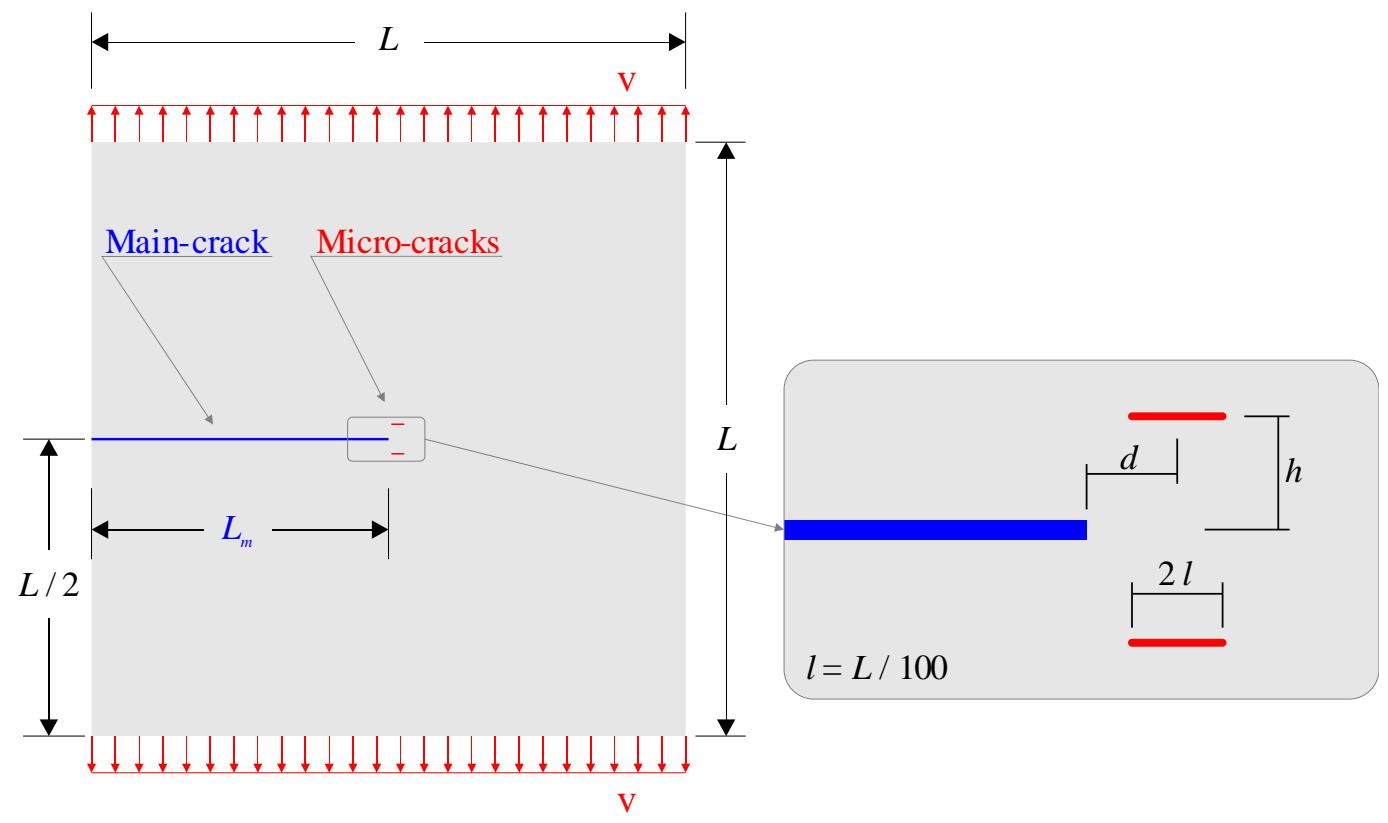

Figure 8 Geometric and parametric details of the benchmark case

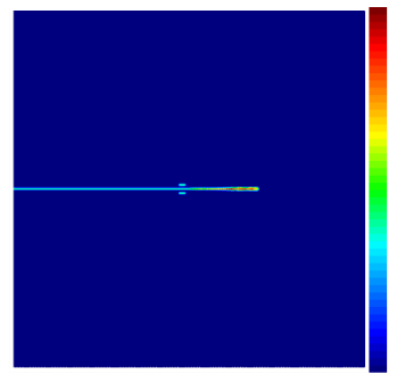

$d / l=-2$

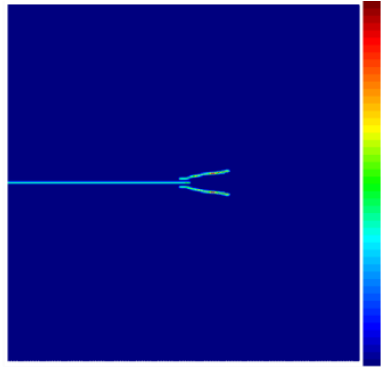

$d / l=0$

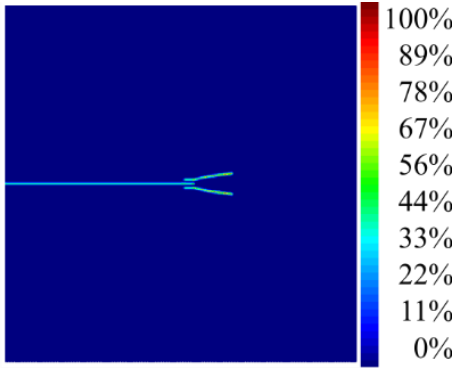

$d / l=2$

Figure 9 Crack propagations with damage $(\varphi)$ percentages for $h / l=1.25$ and related $d / l$ values of the benchmark case at time step 1000

\subsection{Plate with only a main-crack}

In the first place, a case having only the main-crack (Figure 10) is analyzed to determine the propagation behavior without micro-cracks, and to find out the time step at which the complete failure is observed. In the rest of the study, this time step is chosen as a reference step to better understand and evaluate the toughening effects in the presence of various micro-crack formations.

Herein, the crack propagates along the main-crack axis as plotted in Figure 11a. Although some side-crack branches occur after half of the propagation path, the velocity field intensities 
of their tips are not enough to propagate them further (Figure 11b). As a result, it is obtained that the crack starts to propagate approximately at the 500th time step and it reaches to the rightedge almost at the 1700th time step and the complete breakage is realized.

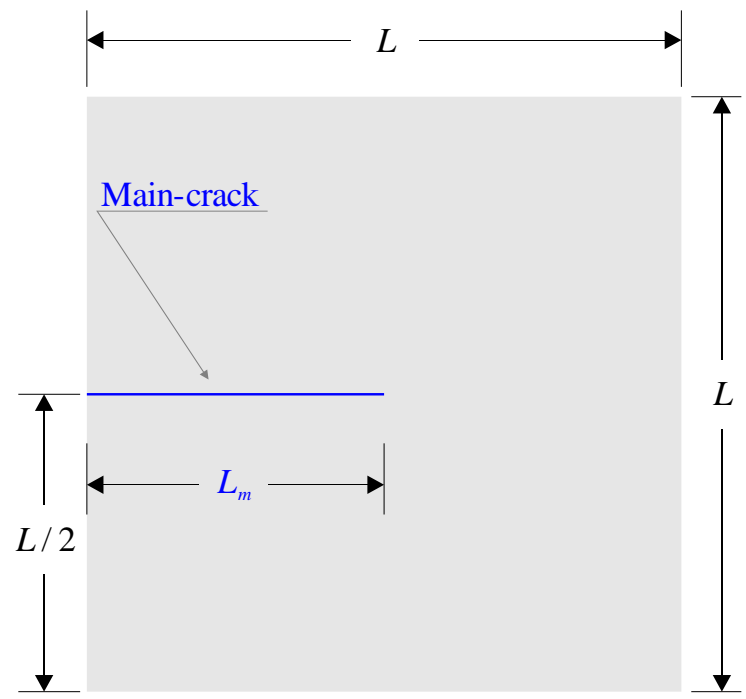

Figure 10 Geometric details of the plate with only a main-crack

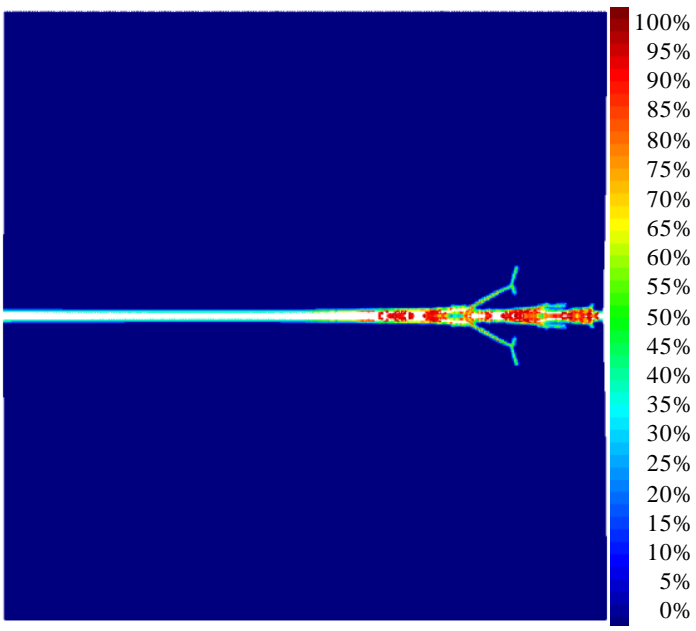

(a)

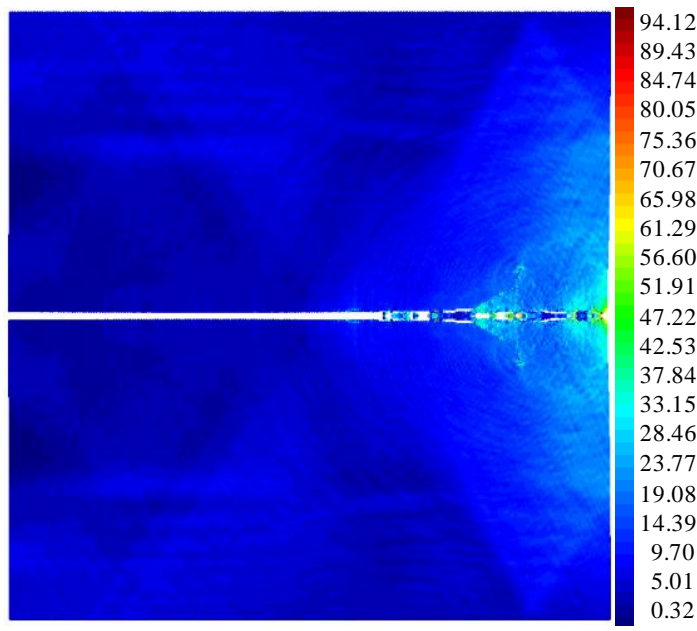

(b)

Figure 11 Plate with only a main-crack at time step 1700: (a) plots of crack propagation with damage $(\varphi)$ percentage; (b) velocity field $(\mathrm{m} / \mathrm{s})$

\subsection{Plate with bifurcated micro-cracks}

In this case, the positioning of the micro-crack cluster is designated to deflect the maincrack from one to the other for more energy dissipation. As depicted in Figure 12, bifurcated micro-cracks are employed to facilitate the branch initiations. Consequently, although the first bifurcated micro-crack located at $2.5 \mathrm{~mm}$ away from the crack tip does not initiate branching, the next one, i.e., located near to the last quarter of the plate, is able to trigger the crack branching (Figure 13a). This bifurcation point is located just before the side-crack branching 
region of the previous case (plate with only a main-crack). Besides, after the first branching, several further side-crack branches occur due to the subsequent micro-cracks. Moreover, the explicit high-velocity values at the rightmost side-crack tips indicate that there may be a further propagation (Figure 13b). Ultimately, there is not any delay in the complete breakage time of the plate despite the occurrence of many branching.

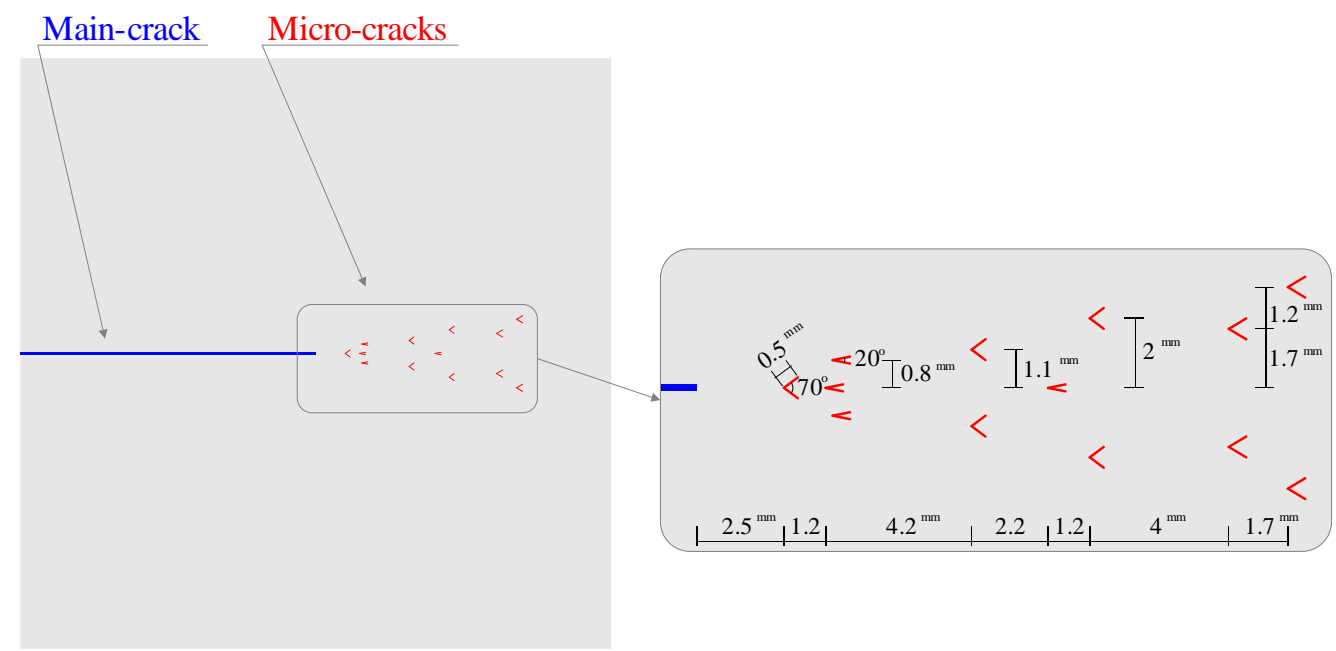

Figure 12 General view and magnified details of micro-cracks in the plate with bifurcated micro-cracks

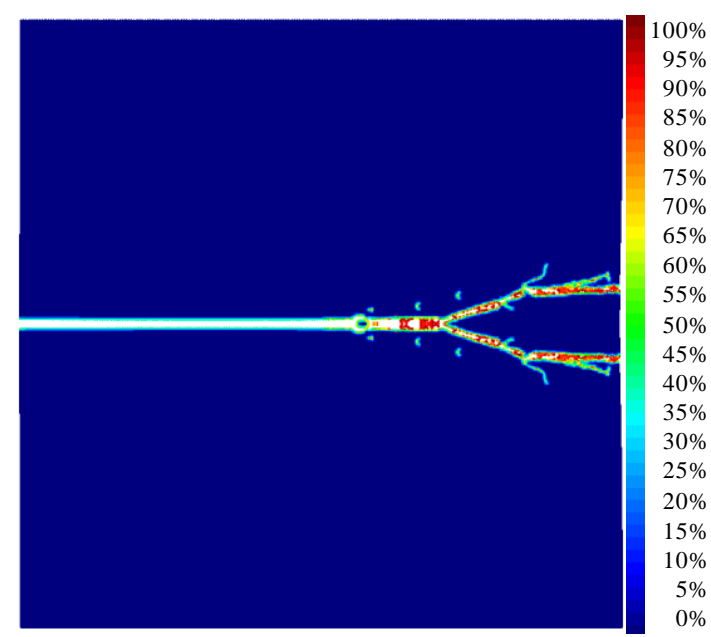

(a)

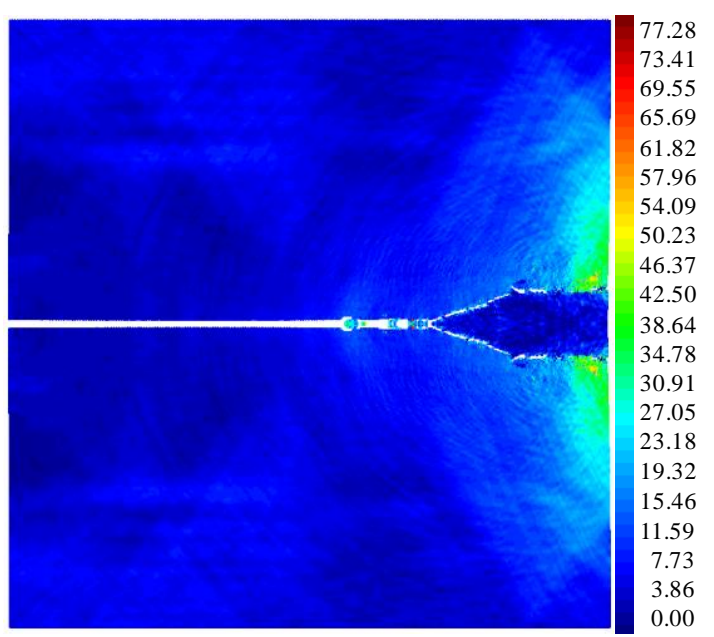

(b)

Figure 13 Plate with bifurcated micro-cracks at time step 1700: (a) plots of crack propagation with damage $(\varphi)$ percentage; (b) velocity field $(\mathrm{m} / \mathrm{s})$

\subsection{Plate with linearly inclined micro-cracks}

Herein, the following arrangements have been added in this case in comparison to the case of the plate with bifurcated micro-cracks: inclined micro-cracks employed, the number of micro-cracks increased, and the micro-crack cluster slightly shifted to the right (Figure 14). Accordingly, the branching occurs when the crack propagation come across with the micro- 
crack cluster, as expected. Then, it follows the path oriented by the cluster (Figure 15a). Moreover, an intensive interaction between micro-cracks is observed with multiple branching of micro-cracks involved in the crack propagation. The energy dissipation in these interactions can be recognized from the velocity field values given in Figure 15b. As a result of these interactions, a multi-fragmented fracture arises. However, although the interactions and the crack-surfaces are significantly increased, the design is insufficient to provide a delay at the complete breakage time of the plate. On the other hand, in this case, the occurred crack-surfaces are considerably more than the previous cases.

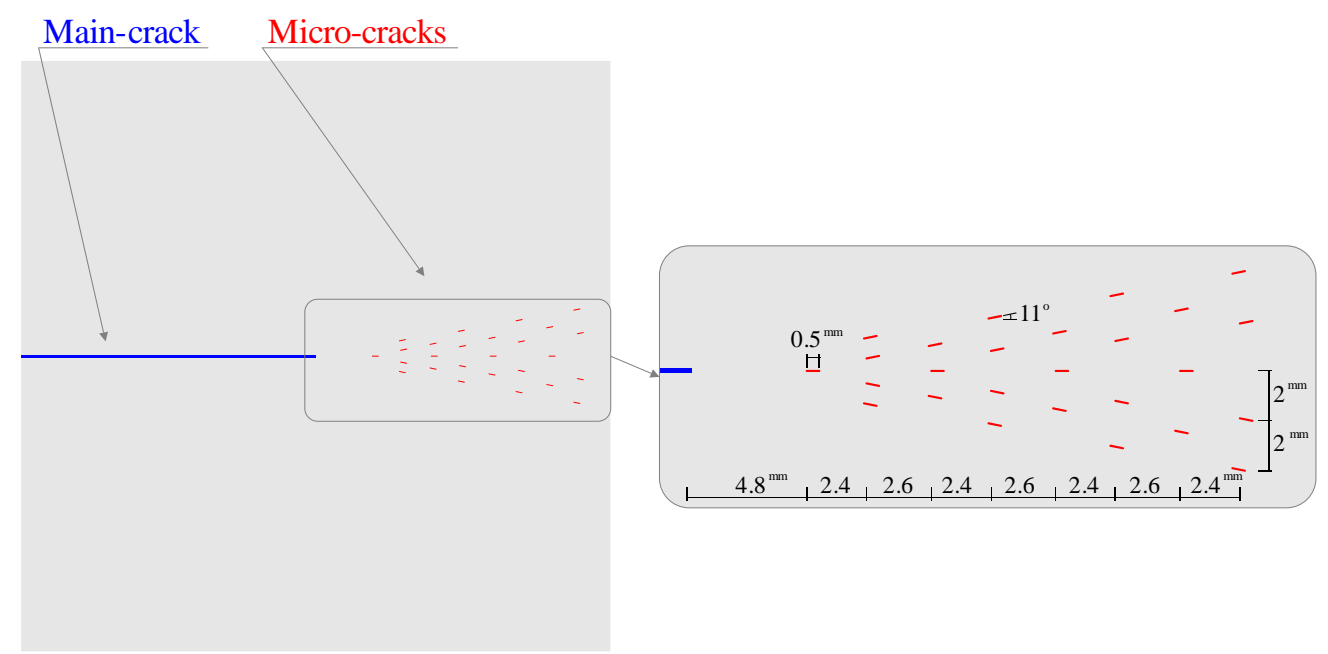

Figure 14 General view and magnified details of micro-cracks in the plate with linearly inclined micro-cracks

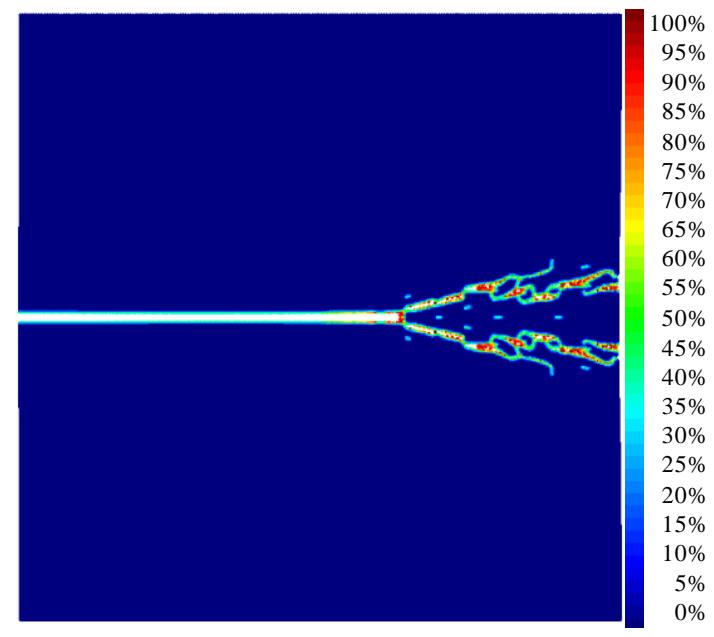

(a)

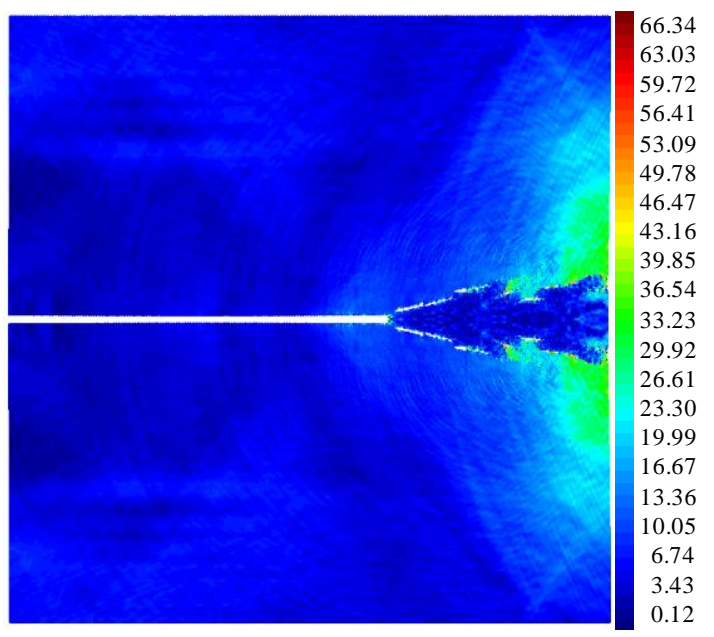

(b)

Figure 15 Plate with linearly inclined micro-cracks at time step 1700: (a) plots of crack propagation with damage $(\varphi)$ percentage; (b) velocity field $(\mathrm{m} / \mathrm{s})$ 


\subsection{Plate with curvilinear and furcated micro-cracks}

In this case, only a furcated micro-crack with five arms, and a curvilinear crack are located at the vicinity of the main-crack tip in contrast to the previous cases where numerous microcracks are employed (Figure 16). The main aim of this example is to initiate two or more crack branches in a point as close as possible to the main-crack tip. Although the expected branching cannot be initiated by the furcated micro-crack, the branching starts almost at the same point as in the bifurcated crack case (Section 3.3) and then two side-crack branches arise as can be seen from Figure 17a. Moreover, the intense velocity values at the side-crack tips indicate that there may be a further propagation (Figure 17b). Hence, there is not any delay in the complete breakage time.

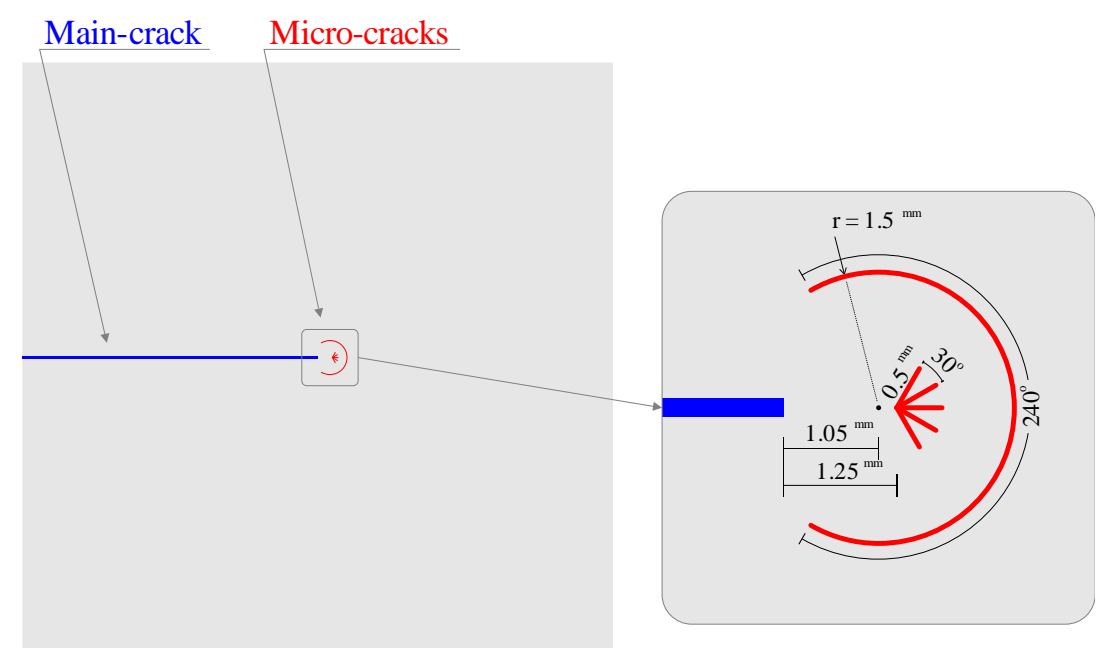

Figure 16 General view and magnified details of micro-cracks in the plate with curvilinear and furcated micro-cracks

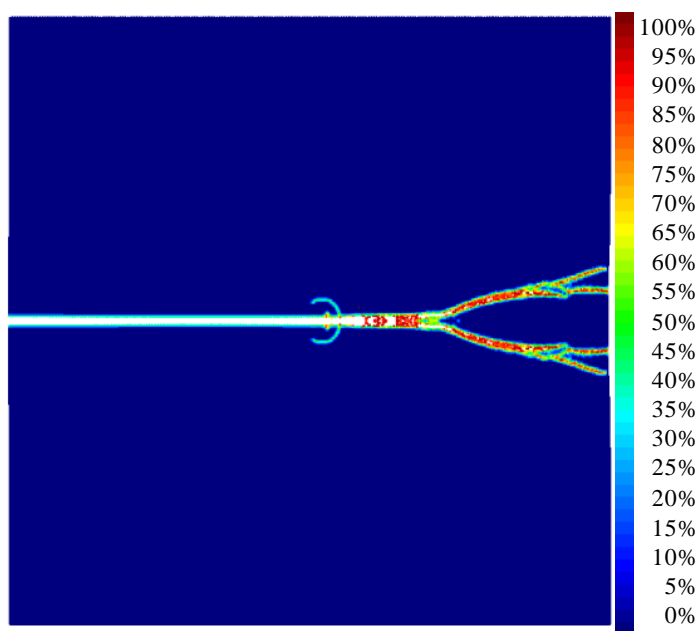

(a)

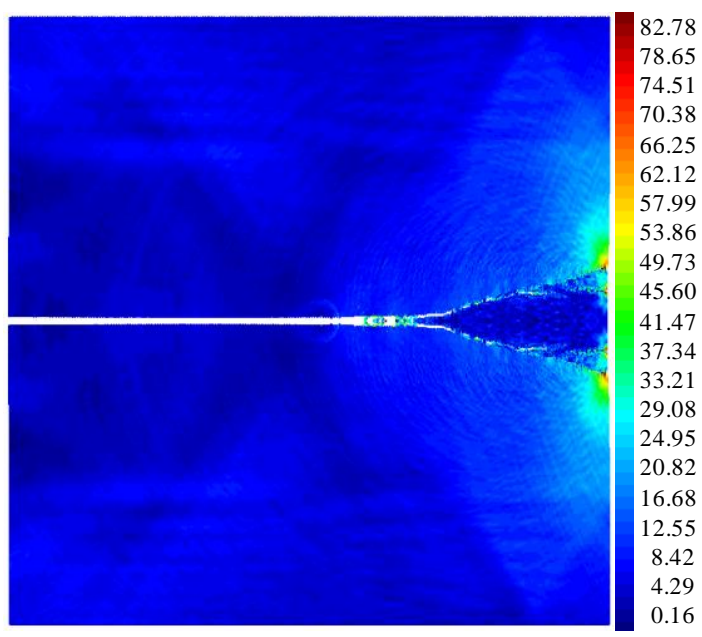

(b)

Figure 17 Plate with curvilinear and furcated micro-cracks at time step 1700: (a) plots of crack propagation with damage $(\varphi)$ percentage; (b) velocity field $(\mathrm{m} / \mathrm{s})$ 


\subsection{Plate with $45^{\circ}$ orbited micro-cracks}

In this case study, the micro-cracks are placed in orbits, centered on the main-crack tip, with the form of a $45^{\circ}$ circle slice. The significant difference between this case and the previous cases is such that the micro-cracks are oriented perpendicular to the crack propagation as depicted in Figure 18. As especially observed in case of the plate with inclined micro-cracks (Section 3.4), the micro-cracks near to the right-edge accelerate the propagation and results in the overall failure of the plate. Therefore, we designed the formation of the micro-crack cluster to be concentrated on the main-crack tip vicinity.

As can be seen from Figure 19a, the crack branching occurs when the crack propagation comes across with the micro-crack cluster and follows the path oriented by the cluster until the third orbit. Then, the propagation continues with a mean $10^{\circ}$ of angle as shown in Figure 19c. Moreover, the velocity intensities on the side-crack branches indicate the ongoing propagation (Figure 19b). In this case, a delay at the complete breakage time is achieved by the shortening total crack path size about $0.9 \%$. This behavior of the crack deflection demonstrates that the orbital allocation of the micro-crack cluster increases the toughness of the material as such the main-crack cannot reach to the right edge of the plate. Overall, this phenomenon is known as material-toughening behavior and herein overserved utilizing bond-based PD implementation.

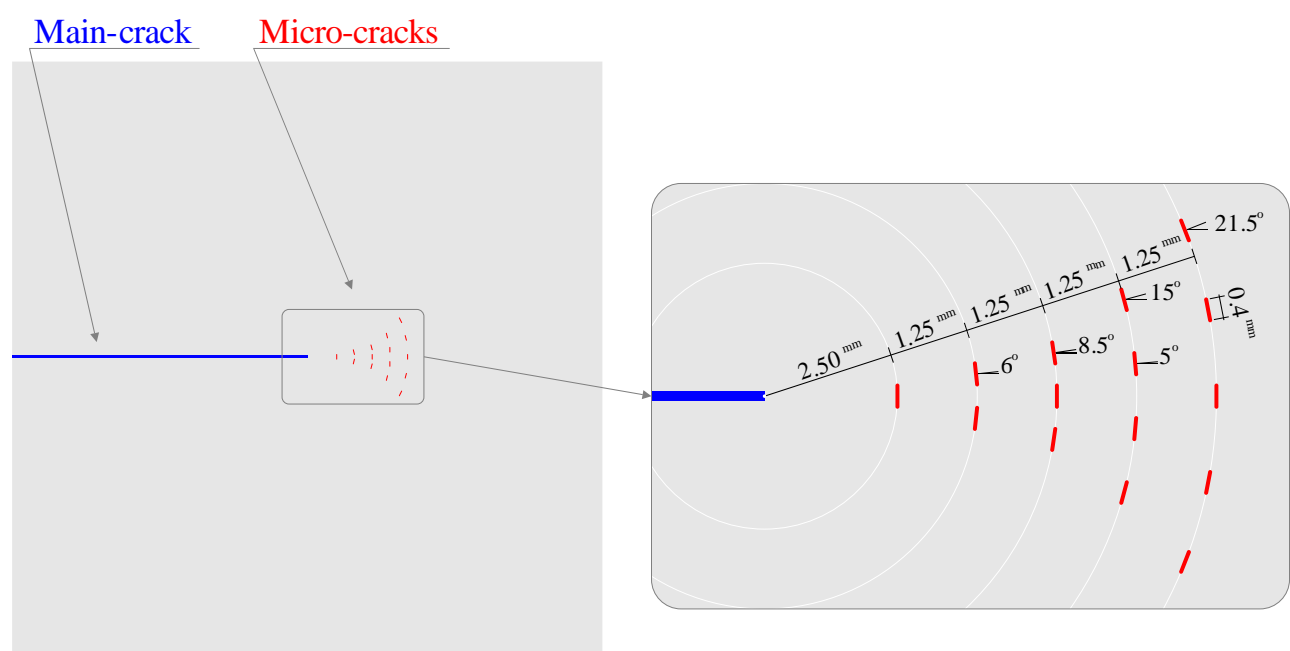

Figure 18 General view and magnified details of micro-cracks in the plate with $45^{\circ}$ orbited micro-cracks 


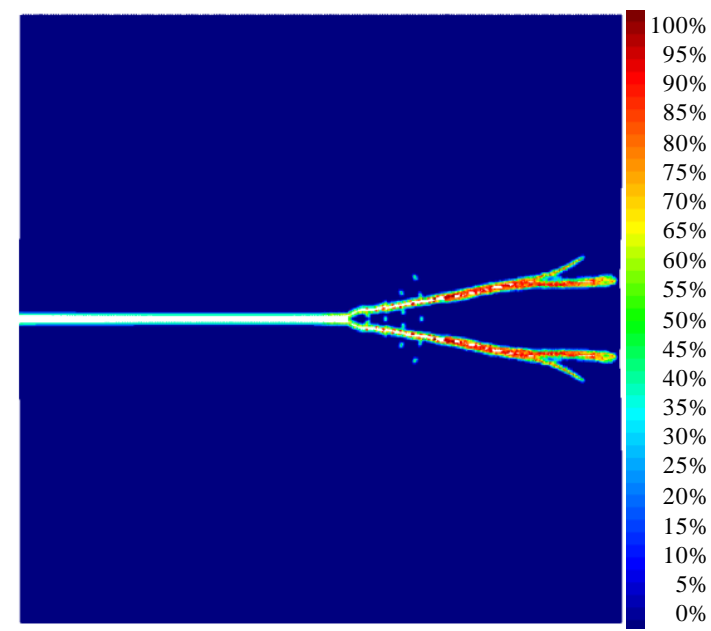

(a)

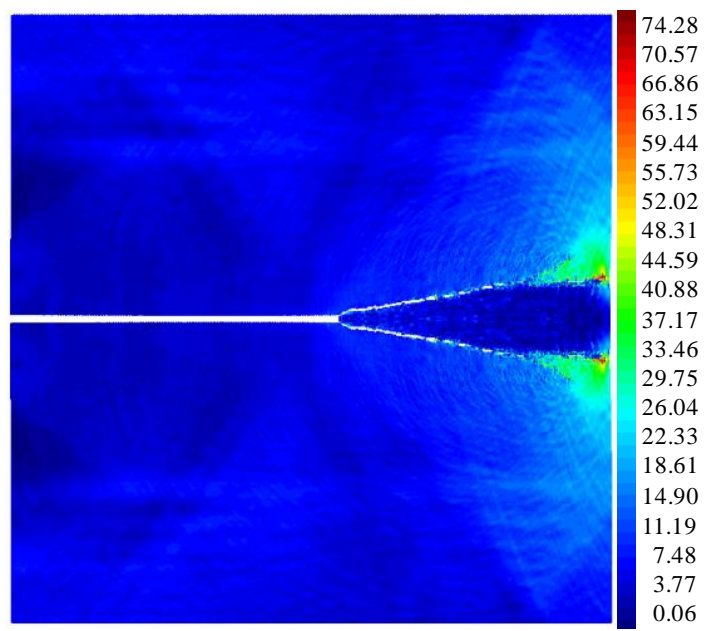

(b)

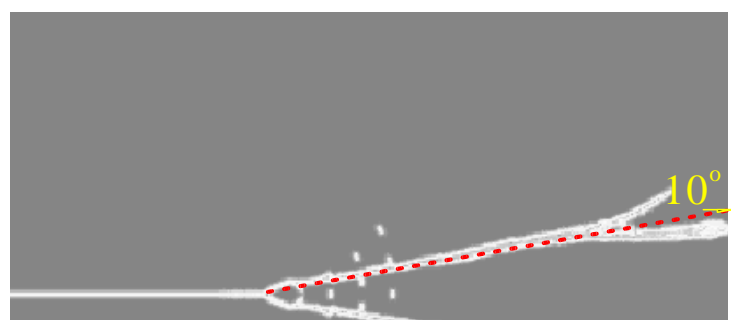

(c)

Figure 19 Plate with $45^{\circ}$ orbited micro-cracks at time step 1700: (a) plots of crack propagation with damage $(\varphi)$ percentage; (b) velocity field $(\mathrm{m} / \mathrm{s})$; (c) mean inclination angle of the major crack branch

\subsection{Plate with $80^{\circ}$ orbited micro-cracks}

To investigate the material-toughening behavior further, the following arrangements have been made in comparison with the previous case: one more orbit added, and the angle of circle slice enlarged to $80^{\circ}$ as presented in Figure 20. Accordingly, the branching occurs when the crack propagation come across with the micro-crack cluster, as expected. Then, it follows the path oriented by the cluster until the fifth orbit as shown in Figure 21a. After that, the propagation continues with a mean $11^{\circ}$ angle (Figure 21c). Moreover, four side-cracks arise in the last quarter of the plate and the velocity intensities on the side-crack branches indicate the ongoing propagation (Figure 21b). Consequently, in this case, a longer delay at the complete breakage time is achieved as compared to one in the previous example (plate with $45^{\circ}$ orbited micro-cracks). Herein, the total crack path size is shortened about $4.5 \%$ of its size, which is five times greater than the previous case. Thus, the orbit angle of the micro-crack cluster has a significant influence on the material-toughening behavior. Hence, it can be inferred that the larger the orbit angle of the micro-cracks is, the tougher the material will become. 


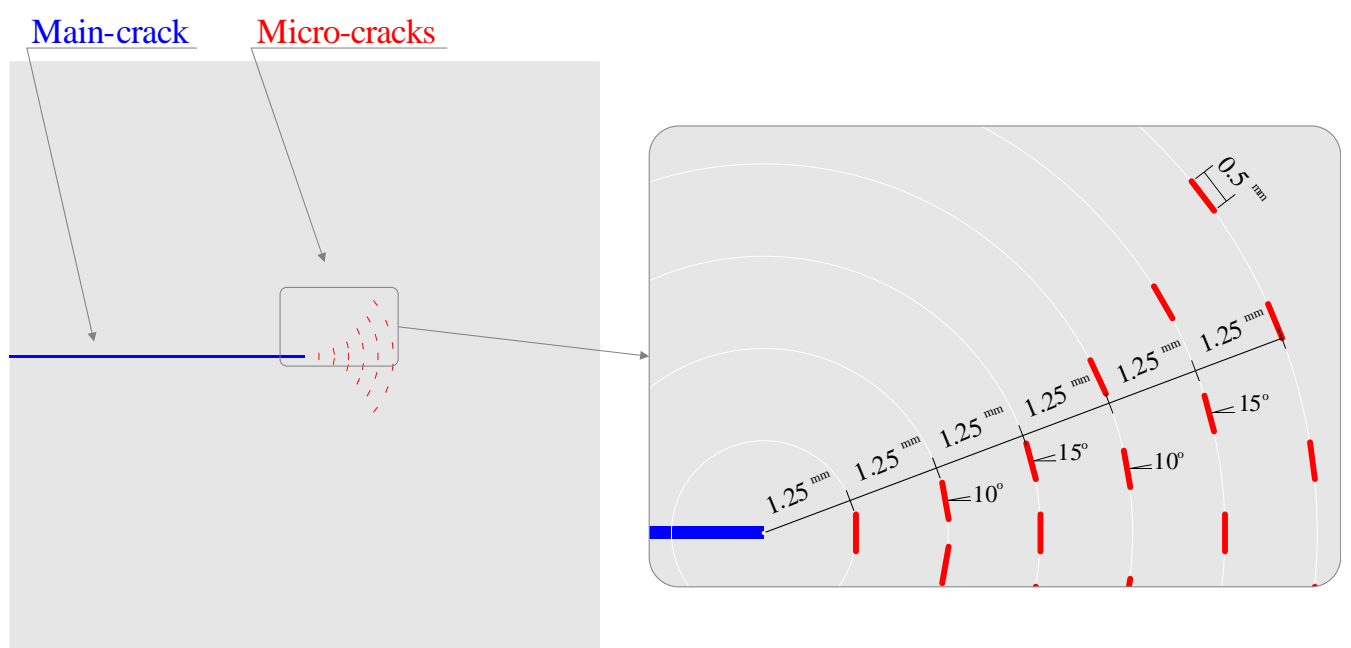

Figure 20 General view and magnified details of micro-cracks in the plate with $80^{\circ}$ orbited micro-cracks

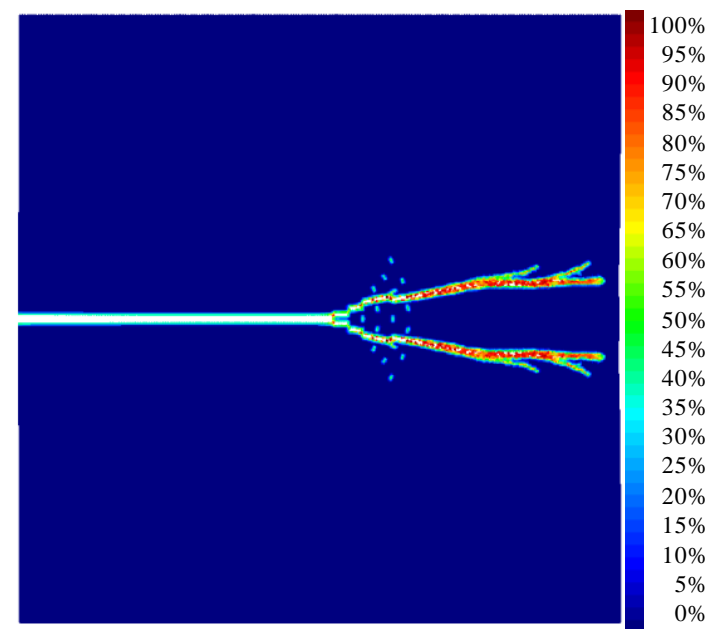

(a)

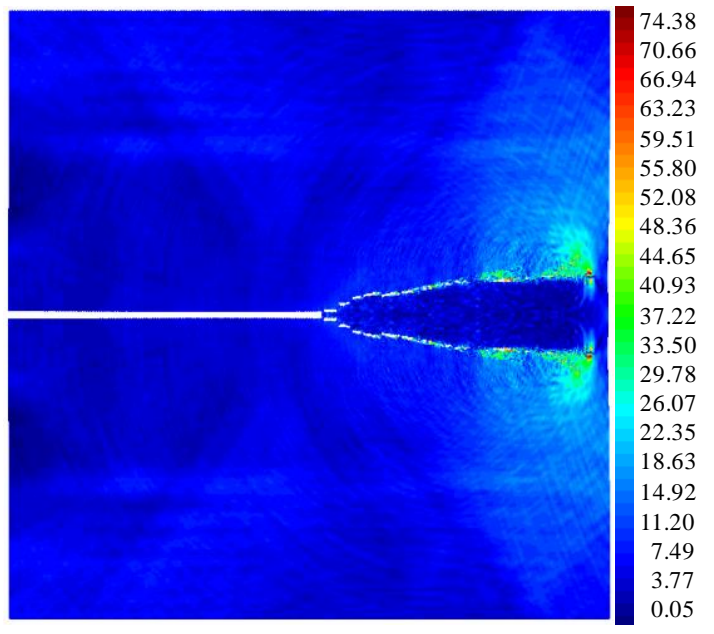

(b)

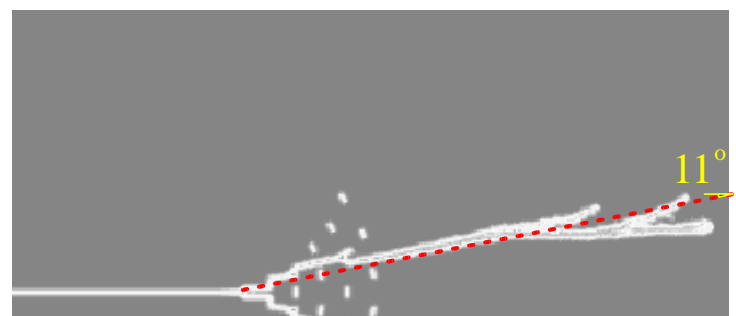

(c)

Figure 21 Plate with $80^{\circ}$ orbited micro-cracks at time step 1700: (a) plots of crack propagation with damage $(\varphi)$ percentage; (b) velocity field $(\mathrm{m} / \mathrm{s})$; (c) mean inclination angle of the major crack branch

\subsection{Plate with a cluster of crescent-like micro-cracks}

In the final case, an extension of the former two cases is made by creating a crescent-like micro-crack cluster where the center of the orbits is moved to the behind of the main-crack tip, and length of micro-cracks are increased almost three times (Figure 22). The main objective 
herein is to reduce the strain energy density at the main-crack tip by encircling it with microcracks. As shown in Figure 23a, the branching occurs when the crack propagation come across with the micro-crack cluster and the propagation continues with a mean $13^{\circ}$ angle (Figure $23 \mathrm{c}$ ). Moreover, four side-crack branches arise in the last quarter of the plate. Besides, the velocity intensities on the side-crack branches indicate the ongoing crack propagation as can be inferred from the red contours presented in Figure 23b. To better understand the physical crack propagation and its evaluation in time, we also provide the percentage of damage occurrence and associated velocity field for different time steps in Figure 24. According to the result contours given in Figure 23-24, a delay at the complete breakage time is achieved even further in this case, as compared to the former two cases (Sections 3.6 and 3.7). In addition, the total size of the crack path is shortened about $7.7 \%$ of its distance, which is almost nine and two times greater than those observed in Sections 3.6 and 3.7, respectively. Hence, among all numerical examples, the most effective branching and toughening occur in this case. Overall, the crescent-like distribution of micro-cracks around the crack tip may be considered as the most material toughening design for materials of the future.

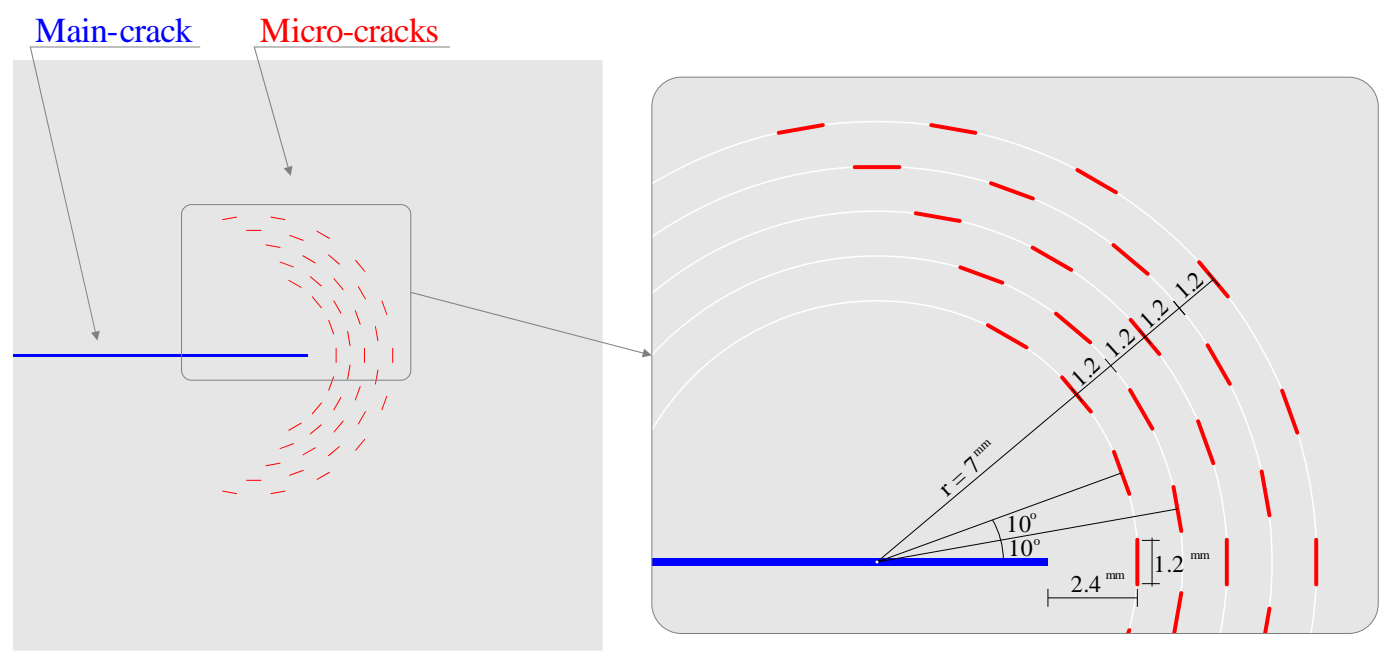

Figure 22 General view and magnified details of micro-cracks in the plate with a cluster of crescent-like micro-cracks 


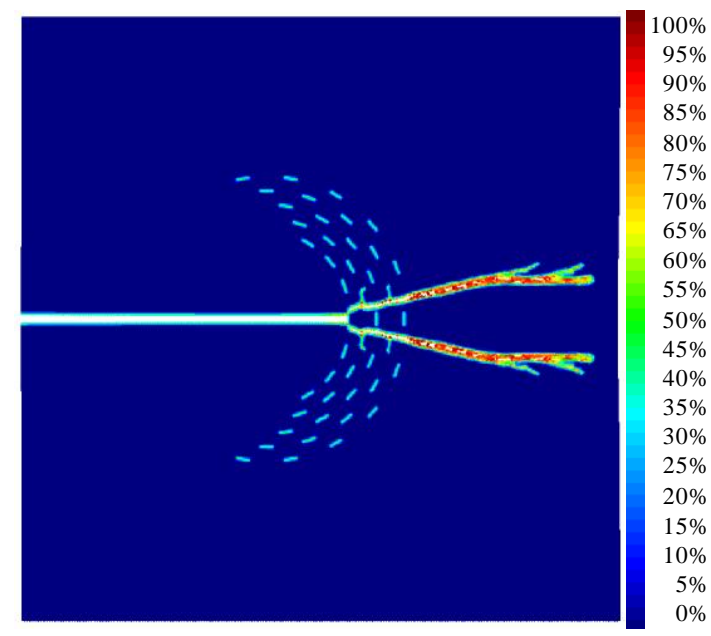

(a)

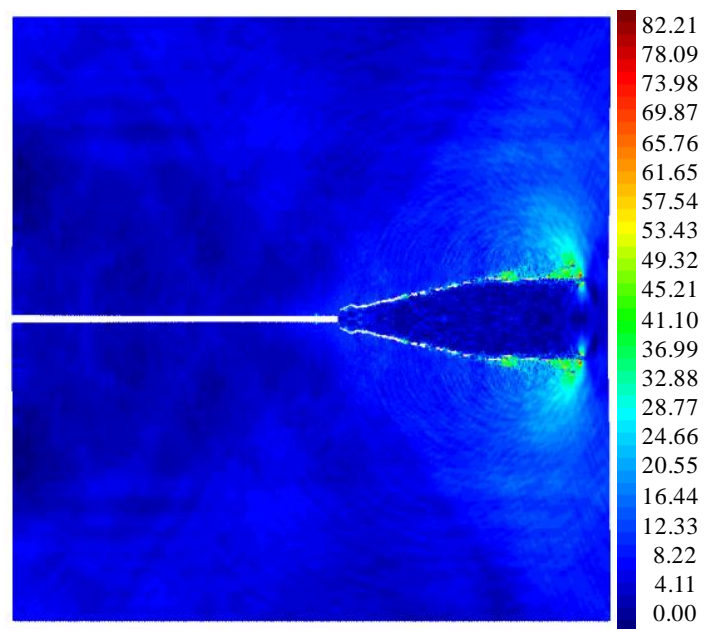

(b)

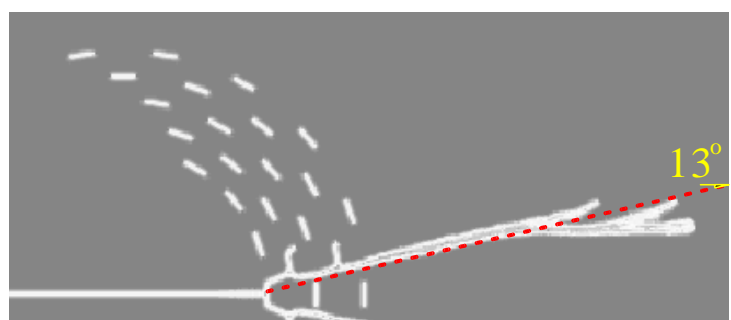

(c)

Figure 23 Plate with a cluster of crescent-like micro-cracks at time step 1700: (a) plots of crack propagation with damage $(\varphi)$ percentage; (b) velocity field $(\mathrm{m} / \mathrm{s})$; (c) mean inclination angle of the major crack branch 


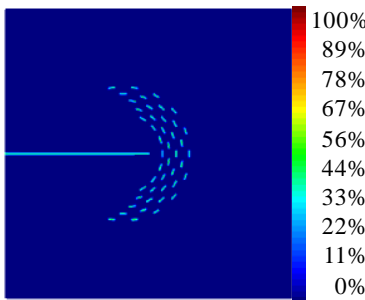

(a)

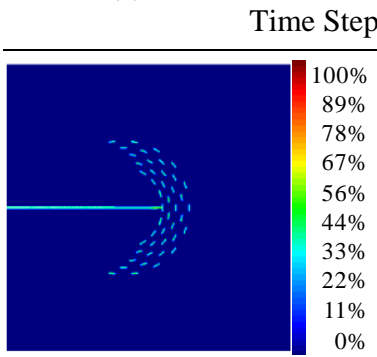

(a)

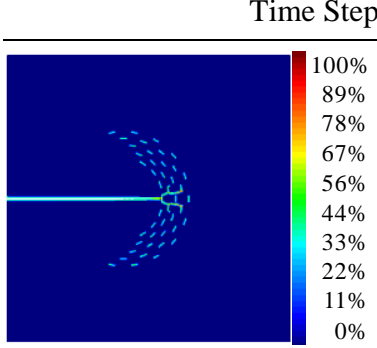

(a)

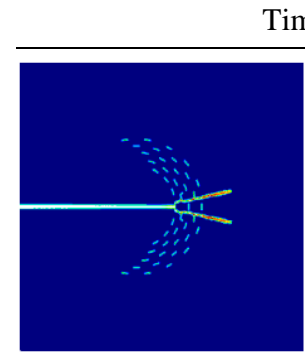

(a)

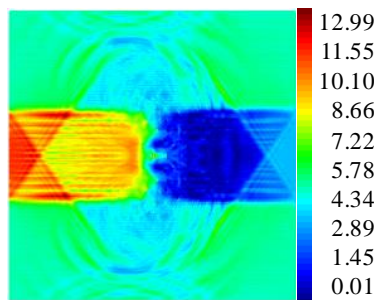

(b)

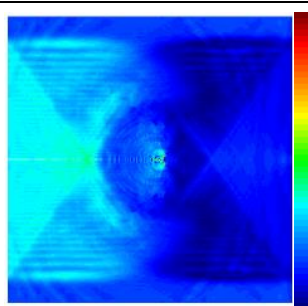

(b)

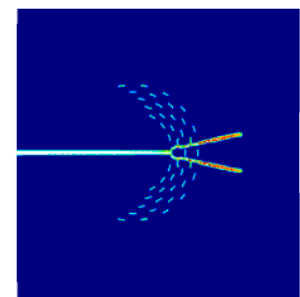

(a)

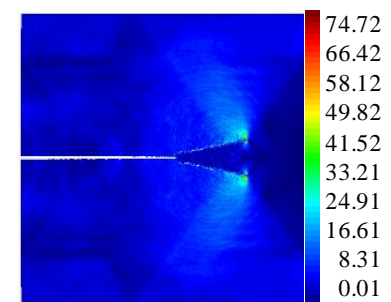

(b)

Time Step: 1400

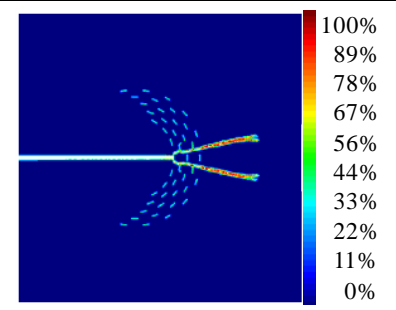

(a)

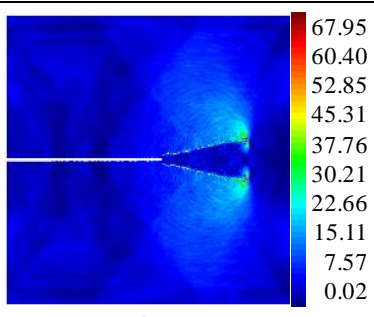

(b)

Time Step: 1500

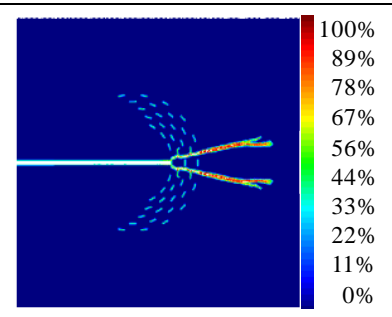

(a)

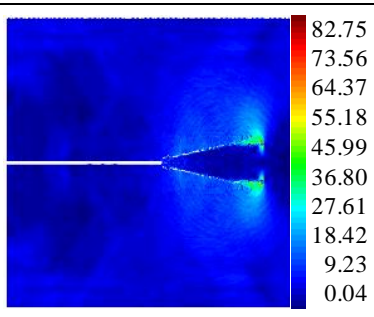

(b)

(b) Time Step: 1600

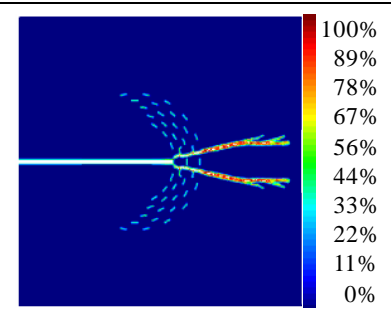

(a)

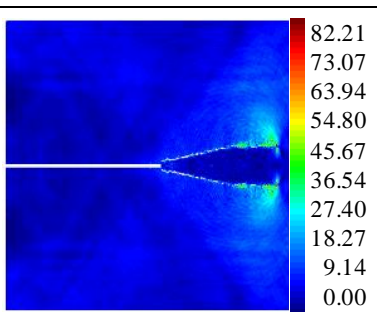

(b)

Time Step: 1300

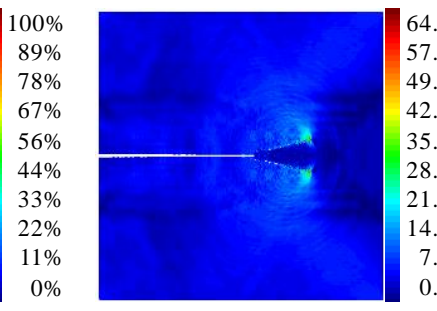

(b)

Figure 24 Plate with a cluster of crescent-like micro-cracks at various time steps: (a) plots of crack propagations with damage $(\varphi)$ percentage; (b) velocity fields $(\mathrm{m} / \mathrm{s})$

\section{Summary of the micro-crack toughening mechanisms}

In an environment containing micro-cracks, the input mechanical energy is used for propagation of the main-crack and, opening and coalescence of the micro-cracks. Dissipating the energy by opening of micro-cracks increases the resistance for the main-crack propagation. This material softening effect around the main-crack tip stabilizes it, hence producing a toughening mechanism. For instance, in the case with crescent-like micro-cracks, it is observed that the most shielding effect was produced due to locating micro-cracks behind the main-crack tip. Also, in the cases with orbited micro-cracks, by increasing the angle the softening enhanced, 
thereby toughening increment was obtained. Hence, the amplification and shielding effects arise depending on the distribution, orientation and spacing of micro-cracks [42].

As detailed in Section 3, various types of micro-crack sizes and orientations are included in the vicinity of the main crack. These micro-crack clusters resulted in different toughening mechanisms for enhanced material design. Herein, we will concisely elaborate on the effects of the inclusion of micro-cracks for providing material toughness through performing quantitative analysis. For this purpose, $x$-coordinate of the propagating main crack, namely moving crack tips, were measured from the center of the plate, i.e., the initial position of the main crack tip before the load is applied. This measurement is made for seven different cases including the case without micro-crack at time steps of $1,500,700,1000$, and from 1300 to 2000 with increments of 100 time steps.

The variations of these $x$-coordinates are plotted versus the time steps in Figure 25. For a fixed time step value, e.g., $1400^{\text {th }}$ step, the positions of the crack can be compared between each specific micro-crack cases. According to this comparison, the more the $x$-coordinate is, the less toughened the material will be. Stated otherwise, the higher the speed of the main crack is, the quicker the failure will be. When the average crack propagation velocities between the $500^{\text {th }}$ and $1600^{\text {th }}$ time steps are calculated as listed in Table 2, the toughening behavior of each case can be observed more explicitly. In Table 2, the velocity of the propagating crack tip reaches its maximum value for the case without any micro-crack, whereas the speed of the main crack takes on the minimum value when the crescent-like micro-cracks are allocated in the vicinity of the main crack. Moreover, the case with curvilinear and furcated micro-cracks produced the higher crack propagation speed than the cases crack with $45^{\circ}$ and $80^{\circ}$ orbited micro-cracks (Table 2). In contrast, the presence the curvilinear and furcated micro-cracks in the plate domain slowed down the speed of the main crack propagation as compared to the presence of the bifurcated micro-cracks and/or linearly inclined micro-cracks (Table 2). 


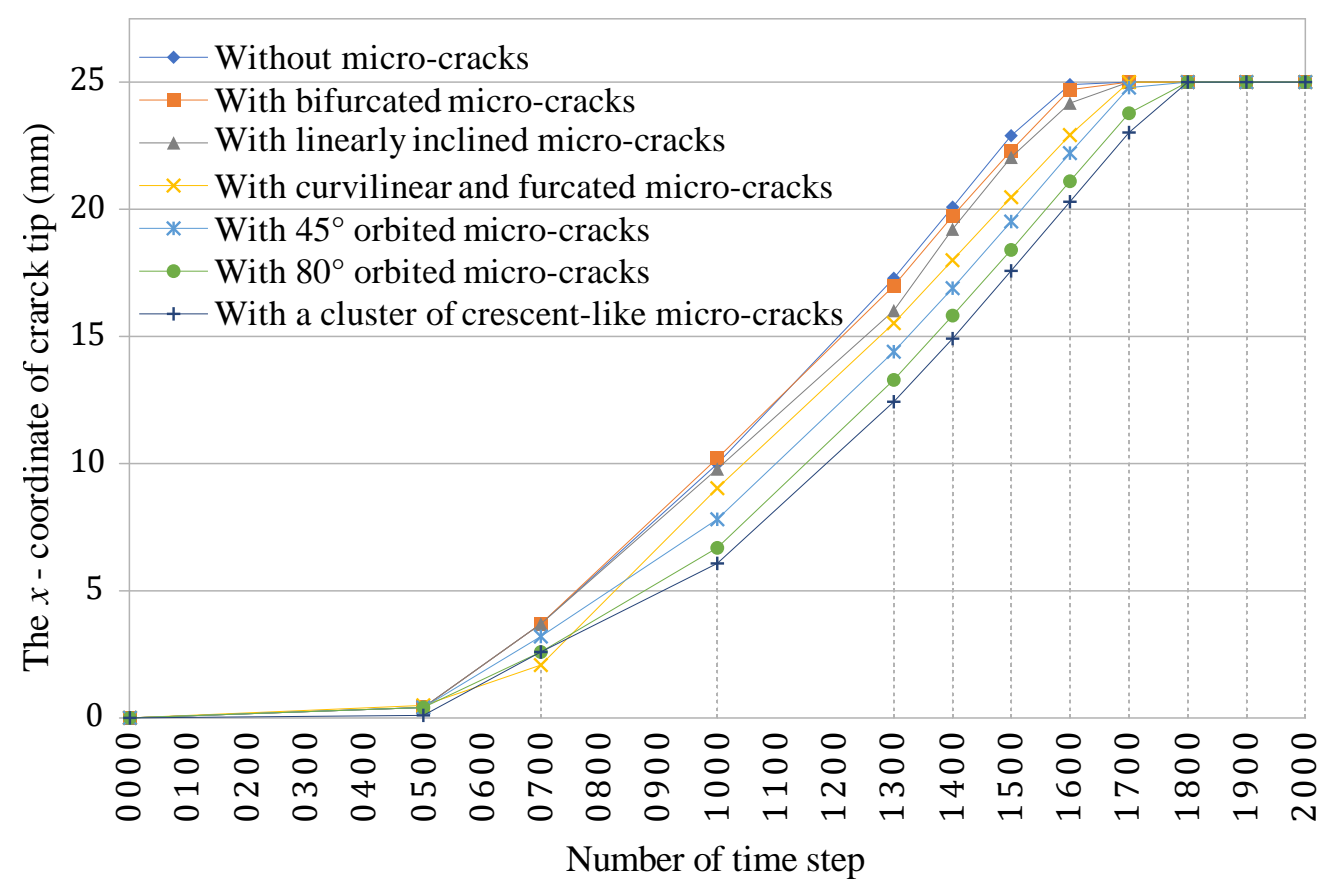

Figure 25 The $x$-coordinates of the propagating main crack tip for all cases versus changing time steps

Table 2 Average velocity of the propagating main crack tip between the $500^{\text {th }}$ and $1600^{\text {th }}$ time steps for all cases

\begin{tabular}{lccc}
\hline & $\begin{array}{c}x \text {-coordinates at } \\
\text { the } 500^{\text {th }} \\
\text { time step } \\
(\mathrm{mm})\end{array}$ & $\begin{array}{c}x \text {-coordinates at } \\
\text { the } 1600^{\mathrm{th}} \\
\text { time step } \\
(\mathrm{mm})\end{array}$ & $\begin{array}{c}\text { Avg. Velocity } \\
\text { between these } \\
\text { time steps } \\
(\mathrm{mm} / \text { time step })\end{array}$ \\
\hline \hline Without micro-cracks & 0,403 & 24,897 & 0,0223 \\
With Bifurcated micro-cracks & 0,403 & 24,696 & 0,0221 \\
With Linearly Inclined micro-cracks & 0,405 & 24,180 & 0,0216 \\
With Curvilinear and Furcated micro-cracks & 0,499 & 22,921 & 0,0204 \\
With $45^{\circ}$ Orbited micro-cracks & 0,404 & 22,210 & 0,0198 \\
With $80^{\circ}$ Orbited micro-cracks & 0,404 & 21,098 & 0,0188 \\
With A Cluster of Crescent-Like micro-cracks & 0,096 & 20,284 & 0,0184 \\
\hline
\end{tabular}

In Figure 25, the dramatic changes in the crack tip positions are noticeably observed between the $1000^{\text {th }}$ and $1700^{\text {th }}$ time steps, thus we select the time steps of 1400,1600 , and 1700 for plotting damage contours in Figure 26. These damage contours confirm the main outcomes of the previous section, as such the $x$-coordinates of the main crack are $20.079 \mathrm{~mm}$ and 14.908 $\mathrm{mm}$ at the time step of 1400 for the cases without micro-crack and with crescent-like microcracks, respectively. According to the decreasing sequence of $x$-coordinates from the first (without micro-crack) to the last (with crescent-like micro-cracks) cases at the $1400^{\text {th }}$ time step, the toughness of the material is increased in the presence of each specific micro-crack pattern 
with the same sequence. Besides, the same material toughing phenomenon is also observed for time steps of 1600 and 1700, as depicted in Figure 26. Overall, these results exhibit the PD computational models developed in this study can be extensively utilized to quantify different toughening mechanism predefined by micro-crack patterns. 


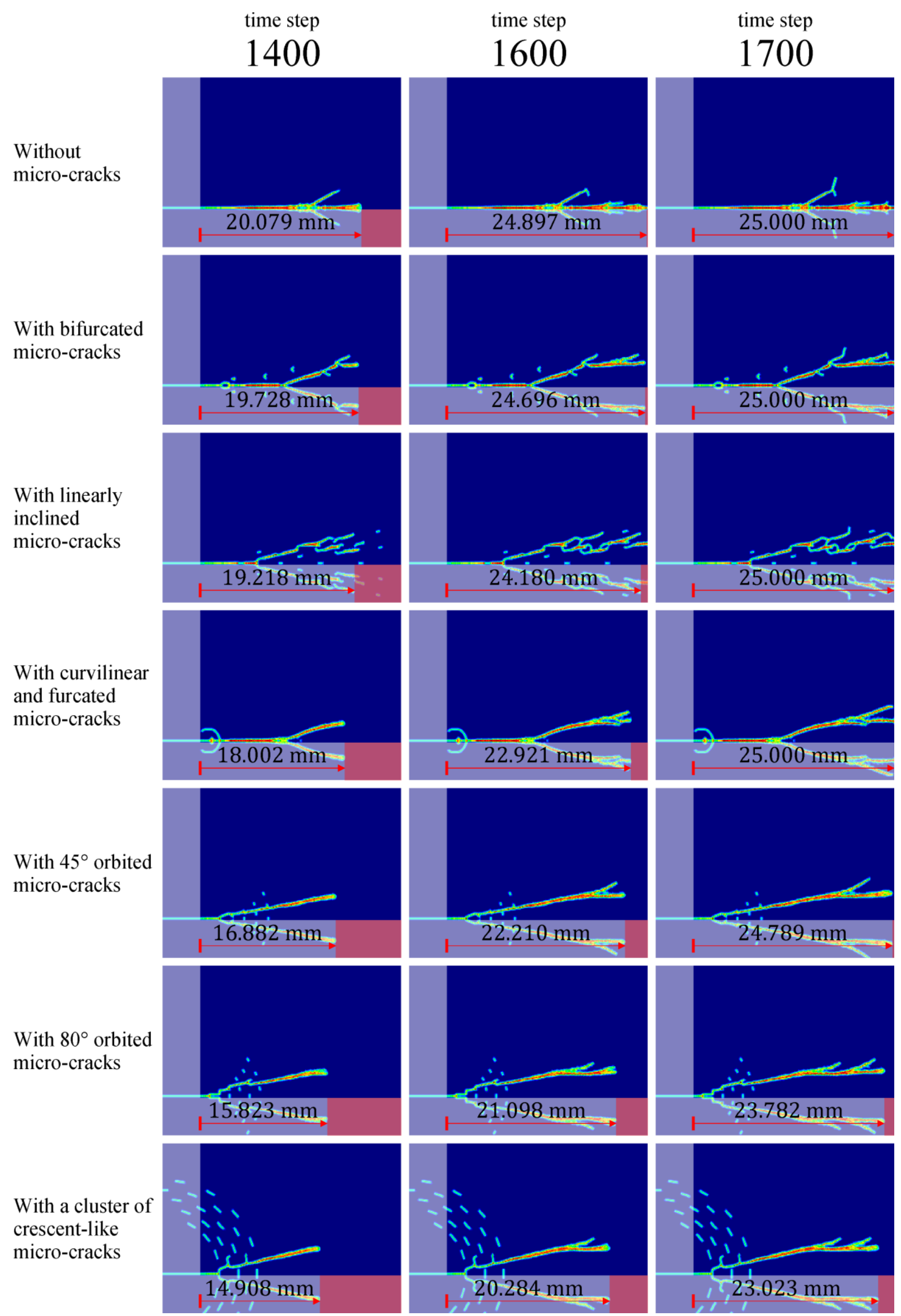

Figure 26 The $x$-coordinates of the propagating main crack tip of all cases at $1400^{\text {th }}, 1600^{\text {th }}$, and $1700^{\text {th }}$ time steps. 


\section{Concluding remarks}

In this study, the former investigation made by Vazic et al. [40] is enhanced by considering more complex micro-crack patterns. With the utilization of bond-based Peridynamic model, the effects of inclined-linear and/or curvilinear micro-crack patterns on dynamic crack propagation are presented for the first time in the literature. It could be deducted from the numerical results that if the branching is initiated as close as possible to the crack tip, the energy dissipation increases, and in turn the complete breakage time could be delayed. Also, increasing the furcation number contributes more energy dissipation. Additionally, the orientation of microcracks is very crucial in design of materials in the presence of pre-defined micro-cracks. In the cases of perpendicularity between main- and micro-cracks the branching and energy dissipation are more effective. As this perpendicularity diminishes and becomes parallel, the main-crack speed boosts. The presented results demonstrate the remarkable capability of Peridynamics in predicting complex fracture patterns formed by a wide range of micro-cracks. With PD theory's capability, the proposed computational models serve to as robust designs of microstructurally toughened new brittle materials. The future directions of this study may include the application of the present idea to the computational design of ductile materials.

\section{Data availability}

The raw/processed data required to reproduce these findings cannot be shared at this time as the data also form part of an ongoing study. 


\section{References}

[1] Loehnert, S. and Belytschko, T. Crack shielding and amplification due to multiple microcracks interacting with a macrocrack. International Journal of Fracture, 145(1), 18 (2007)

[2] Hutchinson, J.W. Crack tip shielding by micro-cracking in brittle solids. Acta Metallurgica, 35(7), 1605-1619 (1987)

[3] Zhou, T., Huang, C., Liu, H., Wang, J., Zou, B. and Zhu, H. Crack propagation simulation in microstructure of ceramic tool materials. Computational Materials Science, 54150-156 (2012)

[4] Li, X., Yang, H., Zan, X., Li, X. and Jiang, X. Effect of a micro-crack on the kinked macro-crack. Theoretical and Applied Fracture Mechanics, 96468-475 (2018)

[5] Bleyer, J., Roux-Langlois, C. and Molinari, J.-F. Dynamic crack propagation with a variational phase-field model: limiting speed, crack branching and velocity-toughening mechanisms. International Journal of Fracture, 204(1), 79-100 (2017)

[6] Brencich, A. and Carpinteri, A. Stress field interaction and strain energy distribution between a stationary main crack and its process zone. Engineering Fracture Mechanics, 59(6), 797-814 (1998)

[7] Li, X., Li, X. and Jiang, X. Influence of a micro-crack on the finite macro-crack. Engineering Fracture Mechanics, 17795-103 (2017)

[8] Xiaotao, L., Xu, L., Hongda, Y. and Xiaoyu, J. Effect of micro-cracks on plastic zone ahead of the macro-crack tip. Journal of Materials Science, 52(23), 13490-13503 (2017)

[9] Rose, L.R.F. Microcrack interaction with a main crack. International Journal of Fracture, 31(3), 233-242 (1986)

[10] Soh, A.K. and Yang, C.H. Numerical modeling of interactions between a macro-crack and a cluster of micro-defects. Engineering Fracture Mechanics, 71(2), 193-217 (2004)

[11] Rubinstein, A.A. Macrocrack interaction with semi-infinite microcrack array. International Journal of Fracture, 27(2), 113-119 (1985)

[12] Kumar, S. and Curtin, W.A. Crack interaction with microstructure. Materials Today, 10(9), 34-44 (2007)

[13] Petrova, V., Tamuzs, V. and Romalis, N. A Survey of Macro-Microcrack Interaction Problems. Applied Mechanics Reviews, 53(5), 117-146 (2000)

[14] Ducourthial, E., Bouchaud, E. and Chaboche, J.L. Influence of microcracks on a propagation of macrocracks. Computational Materials Science, 19(1), 229-234 (2000)

[15] Benedetti, M., Fontanari, V., Monelli, B.D. and Beghini, M. A fully parametric weight function for inclined edge cracks with a kink. Engineering Fracture Mechanics, 136195$212(2015)$

[16] Feng, X.-Q., Li, J.-Y., Ma, L. and Yu, S.-W. Analysis on interaction of numerous microcracks. Computational Materials Science, 28(3), 454-461 (2003)

[17] Xie, Y.J., Li, J., Hu, X.Z., Wang, X.H., Cai, M. and Wang, W. Modelling of multiple crack-branching from Mode-I crack-tip in isotropic solids. Engineering Fracture Mechanics, 109105-116 (2013)

[18] Zhou, X., Wang, Y. and Qian, Q. Numerical simulation of crack curving and branching in brittle materials under dynamic loads using the extended non-ordinary state-based peridynamics. European Journal of Mechanics - A/Solids, 60277-299 (2016)

[19] Wang, H., Liu, Z., Xu, D., Zeng, Q. and Zhuang, Z. Extended finite element method analysis for shielding and amplification effect of a main crack interacted with a group of nearby parallel microcracks. International Journal of Damage Mechanics, 25(1), 4-25 (2016) 
[20] Budyn, É., Zi, G., Moës, N. and Belytschko, T. A method for multiple crack growth in brittle materials without remeshing. International Journal for Numerical Methods in Engineering, 61(10), 1741-1770 (2004)

[21] Moës, N., Dolbow, J. and Belytschko, T. A finite element method for crack growth without remeshing. International Journal for Numerical Methods in Engineering, 46(1), 131-150 (1999)

[22] Zhuang, Z. and Cheng, B.-B. Development of X-FEM methodology and study on mixedmode crack propagation. Acta Mechanica Sinica, 27(3), 406-415 (2011)

[23] Zhuang, Z. and Cheng, B.B. Equilibrium state of mode-I sub-interfacial crack growth in bi-materials. International Journal of Fracture, 170(1), 27-36 (2011)

[24] Zhuang, Z. and Cheng, B. A novel enriched CB shell element method for simulating arbitrary crack growth in pipes. Science China Physics, Mechanics and Astronomy, 54(8), 1520-1531 (2011)

[25] Bobaru, F. and Zhang, G. Why do cracks branch? A peridynamic investigation of dynamic brittle fracture. International Journal of Fracture, 196(1), 59-98 (2015)

[26] Silling, S.A. and Askari, E. A meshfree method based on the peridynamic model of solid mechanics. Computers and Structures, 83(17-18), 1526-1535 (2005)

[27] Silling, S.A. Reformulation of elasticity theory for discontinuities and long-range forces. Journal of the Mechanics and Physics of Solids, 48(1), 175-209 (2000)

[28] Silling, S.A., Epton, M., Weckner, O., Xu, J. and Askari, E. Peridynamic states and constitutive modeling. Journal of Elasticity, 88(2), 151-184 (2007)

[29] Silling, S.A. and Lehoucq, R.B. Convergence of Peridynamics to Classical Elasticity Theory. Journal of Elasticity, 93(1), 13 (2008)

[30] Oterkus, E. and Madenci, E. Peridynamic Analysis of Fiber-Reinforced Composite Materials. Journal of Mechanics of Materials and Structures, 7(1), 45-84 (2012)

[31] Ghajari, M., Iannucci, L. and Curtis, P. A peridynamic material model for the analysis of dynamic crack propagation in orthotropic media. Computer Methods in Applied Mechanics and Engineering, 276431-452 (2014)

[32] Oterkus, E., Madenci, E., Weckner, O., Silling, S., Bogert, P. and Tessler, A. Combined finite element and peridynamic analyses for predicting failure in a stiffened composite curved panel with a central slot. Composite Structures, 94(3), 839-850 (2012)

[33] Kilic, B. and Madenci, E. Coupling of Peridynamic Theory and the Finite Element Method. Journal of Mechanics of Materials and Structures, 5(5), 707-733 (2010)

[34] De Meo, D., Diyaroglu, C., Zhu, N., Oterkus, E. and Siddiq, M.A. Modelling of stresscorrosion cracking by using peridynamics. International Journal of Hydrogen Energy, 41(15), 6593-6609 (2016)

[35] Askari, E., Bobaru, F., Lehoucq, R.B., Parks, M.L., Silling, S.A. and Weckner, O. Peridynamics for multiscale materials modeling. Journal of Physics: Conference Series, 125(1), 012078 (2008)

[36] Oterkus, S., Madenci, E. and Agwai, A. Fully coupled peridynamic thermomechanics. Journal of the Mechanics and Physics of Solids, 641-23 (2014)

[37] Oterkus, S., Madenci, E. and Agwai, A. Peridynamic thermal diffusion. Journal of Computational Physics, 26571-96 (2014)

[38] Madenci, E. and Oterkus, S. Ordinary state-based peridynamics for plastic deformation according to von Mises yield criteria with isotropic hardening. Journal of the Mechanics and Physics of Solids, 86192-219 (2016)

[39] Madenci, E. and Oterkus, E. Peridynamic Theory and Its Applications, Springer, New York (2014) 
[40] Vazic, B., Wang, H., Diyaroglu, C., Oterkus, S. and Oterkus, E. Dynamic propagation of a macrocrack interacting with parallel small cracks. AIMS Materials Science, 4(1), 118136 (2017)

[41] Ha, Y.D. and Bobaru, F. Studies of dynamic crack propagation and crack branching with peridynamics. International Journal of Fracture, 162(1), 229-244 (2010)

[42] Rao, G.M.N. and Murthy, C.R. Dual role of microcracks: toughening and degradation. Canadian Geotechnical Journal, 38(2), 427-440 (2001) 